\title{
Soft gamma-ray sources detected by INTEGRAL
}

\author{
D. Petry ${ }^{1,2,4}$, V. Beckmann ${ }^{2,3}$, H. Halloin ${ }^{3}$, and A. Strong ${ }^{1}$ \\ 1 Max Planck Institute for extraterrestrial Physics (MPE), Giessenbachstr., 85748 Garching, Germany \\ e-mail: dpetry@eso.org \\ 2 ISDC Data Centre for Astrophysics, Ch. d'Ecogia 16, 1290 Versoix, Switzerland \\ 3 APC, UMR 7164, Université Paris 7 Denis Diderot, 10 rue Alice Domon et Léonie Duquet, 75025 Paris Cedex 13, France \\ ${ }^{4}$ now at European Southern Observatory, Karl-Schwarzschild-Str. 2, 85748 Garching, Germany
}

Received 18 July 2009 / Accepted 2 September 2009

\section{ABSTRACT}

\begin{abstract}
Aims. We aim to exploit the available INTEGRAL/SPI data to provide time-averaged spectra of the brightest soft gamma-ray sources Methods. Employing a maximum-likelihood fit technique for our SPI data analysis, we take as input to our source model the source catalog derived by Bouchet et al. (2008) from a SPI all-sky study. We use the first four years of public SPI data and extract spectra between $25 \mathrm{keV}$ and $1 \mathrm{MeV}$ for the 20 catalog sources detected by Bouchet et al. at 200-600 keV with $\geq 2.5 \sigma$. In order to verify our analysis, we also extract spectra for the same sources from the corresponding INTEGRAL/ISGRI data. We fit adequate spectral models to the energy range $25-1000 \mathrm{keV}$ for SPI and 25-600 keV for ISGRI. We use our spectra from the Crab (which is among the 20 sources studied here) and an empty location in a crowded field to derive an estimation of the systematic errors.

Results. The agreement between our SPI and ISGRI measurements is good if we normalise them on the Crab spectrum. Our SPI flux measurements also agree well with those by Bouchet et al. (2008). All 20 sources in our sample are detected independently in the bands $25-100 \mathrm{keV}$ and $100-200 \mathrm{keV}$. At $200-600 \mathrm{keV}$ we detect eight sources, at $600-1000 \mathrm{keV}$ we detect two sources. Our spectra agree well with the results from previous publications where available. For six of the 14 XRBs in our sample we find evidence for a hard powerlaw-component which becomes dominant above the cutoff energy of the thermal Comptonization component. In two of these cases, our study provides the first indication of such emission. For the others, our results confirm previous studies. Our spectrum of the Crab, integrated over $1.3 \mathrm{Ms}$, shows a significant flux in all points and is well described by a powerlaw with a break near $100 \mathrm{keV}$ and spectral indices 2.11 and 2.20.
\end{abstract}

Key words. gamma rays: observations $-\mathrm{X}$-rays: binaries

\section{Introduction}

Soft gamma-rays have to be observed with satellite-based instruments, and so far there have been only three major missions taking data in the range from $100 \mathrm{keV}$ to a few $\mathrm{MeV}$ with adequate sensitivity: GRANAT with the SIGMA instrument (Revnivtsev et al. 2004), CGRO with OSSE (Johnson et al. 1993) and COMPTEL (Schönfelder et al. 2000), and INTEGRAL with IBIS (Ubertini et al. 2003), and SPI (Vedrenne et al. 2003).

On INTEGRAL, the only soft gamma-ray observatory operational today, both the high-energy instruments, IBIS and SPI, are capable of measuring point source spectra. They complement each other in that IBIS provides angular resolution and good sensitivity at energies below a few $100 \mathrm{keV}$ while SPI has superior energy resolution and high-energy sensitivity and permits the study of extended diffuse emission with its large field of view.

Sources of soft gamma radiation with photon energies between $100 \mathrm{keV}$ and several $\mathrm{MeV}$ are astrophysically interesting because they permit us to study matter at supra-thermal energies. Essentially all known point-like emitters of such radiation consist of compact objects such as black holes or neutron stars which convert the gravitational energy of their surrounding matter or their own extreme rotational and magnetic energy into translational kinetic energy and then in turn partially into highenergy photons. The black holes come either as stellar black holes in X-ray binaries or as supermassive black holes in the centres of galaxies. All other sources of soft gamma-rays are spatially extended or unresolved and high in number-density. They form a continuum which seems, in the case of our Galaxy, to extend over the bulge and a significant part of the disk (Bouchet et al. 2005; Revnivtsev et al. 2006; Krivonos et al. 2007).

In the most recent all-sky study of INTEGRAL/SPI soft gamma-ray data, Bouchet et al. (2008) have presented a detailed analysis of both diffuse and point source emission in the energy range from $25 \mathrm{keV}$ to $600 \mathrm{keV}$ based on all SPI data from February 2003 up to May 2006. They conclude that the diffuse component becomes more prominent with increasing energy reaching $32 \%$ in the central radian above $100 \mathrm{keV}$. The diffuse emission is bright over up to $\pm 45^{\circ}$ in galactic longitude and $\pm 10^{\circ}$ in galactic latitude depending on energy.

Bouchet et al. (2008), B08 in the following, also present separate catalogs of point sources for the four energy ranges 25-50 keV, 50-100 keV, 100-200 keV, and 200-600 keV which they detect at least at a significance level $\geq 2.5 \sigma$. As the sensitivity of SPI relative to the steeply dropping source spectra decreases with increasing energy and the diffuse emission becomes more dominant, the number of detected point sources decreases with increasing energy as well. In the energy range 200-600 keV a total of 20 sources are detected.

In this work, we take the B08 catalog for the energy range 200-600 keV and derive time-averaged INTEGRAL/SPI spectra for each of the 20 point sources contained in it. The resulting catalog of spectra of the 20 brightest soft gamma-ray sources 
will be useful to, among others, modellers of the hard X-ray and soft gamma-ray background caused by unresolved sources of the same source classes as in our catalog, high-energy observers of these sources who have to average over longer time-scales in order to achieve detections with acceptable significance, and generally researchers interested in the energy balance of the galaxy (most of the 20 sources are galactic).

We employ a newly developed maximum likelihood fit analysis technique based on the spimodfit software by Halloin \& Strong (2007) to all public INTEGRAL data available for these sources (i.e. up to November 2006, the status when our dataset was frozen). In order to minimise inhomogeneities of our systematic uncertainties, the analysis method is not optimised for any individual source, in particular not for the Crab.

For comparison and verification of our method, we also derive the spectra for the same sources from the corresponding INTEGRAL/IBIS/ISGRI data.

This paper is meant to serve two purposes: (a) be a supplement to B08 and investigate the average spectra of the brightest known soft gamma-ray sources; and (b) demonstrate an analysis technique for INTEGRAL/SPI data based on spimodfit.

In Sect. 2, the SPI and ISGRI instruments are briefly introduced, and the observations and the data selection are described. Section 3 describes the analysis technique. Section 4 presents and discusses the spectra individually while Sect. 5 summarises the results, discusses them jointly and concludes.

\section{Instruments and observations}

This study uses data from the SPI and the ISGRI instruments on INTEGRAL. The properties of these instruments are discussed in detail elsewhere (see Vedrenne et al. (2003) and Roques et al. (2003) for SPI and Ubertini et al. (2003) for ISGRI). Here we just briefly summarise the main aspects.

The ISGRI instrument is part of the high-sensitivity codedmask imager IBIS. It has a fully coded field of view of $9^{\circ} \times 9^{\circ}$, an angular resolution of $12^{\prime} F W H M$, and a spectral resolution of $9 \%$ at $100 \mathrm{keV}$. Its well calibrated energy range is $14 \mathrm{keV}$ to ca. $700 \mathrm{keV}$. The nominal continuum sensitivity $(\Delta E=E / 2,3 \sigma$ in $\left.10^{5} \mathrm{~s}\right)$ at $100 \mathrm{keV}$ is $3 \mathrm{mCrab}$.

The SPI instrument is the coded-mask imaging highresolution spectrometer on INTEGRAL. Its fully coded field of view has a radius of $8^{\circ}$. With its 19 pixels (hexagonal, cooled Ge detectors) it achieves an angular resolution of $2.5^{\circ} \mathrm{FWHM}$. The average spectral resolution of the detectors deteriorates only marginally as a function of energy from $\Delta E \approx 1.8 \mathrm{keV}$ below $200 \mathrm{keV}$ to $\Delta E \approx 4 \mathrm{keV}$ at $3 \mathrm{MeV}$. This means, the relative energy resolution improves from $0.9 \%$ at $200 \mathrm{keV}$ to $0.13 \%$ above $3 \mathrm{MeV}$. The calibrated energy range is $25-8000 \mathrm{keV}$. The nominal continuum sensitivity (as defined above for IBIS) at $100 \mathrm{keV}$ is $16 \mathrm{mCrab}$. But above $1 \mathrm{MeV}$, the continuum sensitivity for point sources becomes inadequate. Only the Crab is detected at 1-8 MeV, and an electronic noise problem between 1 and $2 \mathrm{MeV}$ results in large systematic errors in this interesting energy range. We therefore limit this study to the range $25-1000 \mathrm{keV}$.

Both energy resolution and sensitivity are time dependent: radiation damage degenerates the detectors leading to a deterioration of the energy resolution by a few tenths of a keV (negligible for continuum analysis) over timescales of months. This degradation is cured by regular annealings (controlled heatings) of the detectors. An annealing period lasts typically 12 days during which no science data can be taken.

While the spectral resolution is hence kept constant for our purposes, the sensitivity of SPI remains time dependent because the continued exposure to cosmic radiation leads to increasing background from the activation of all parts of the INTEGRAL satellite. In addition, the intensity of the cosmic radiation itself is strongly time dependent because of solar wind variablity (solar cycle) and solar flares. Over the course of the INTEGRAL mission so far, this has lead to a nearly steady increase in instrumental background. In November 2006, the instrumental background had increased by about $80 \%$ compared to the time shortly after launch (November 2002). It is expected that this trend will not continue (and possibly be reversed) beyond the end of the present solar minimum which has been a very prolonged one.

\subsection{Data selection}

The INTEGRAL data, as they are delivered to scientists, are subdivided into time periods of constant pointing, so-called science windows which typically have a duration of about $30 \mathrm{~min}$. A second relevant time unit is the "revolution" or orbit which is important to the science analysis because the highly excentric orbit of the INTEGRAL spacecraft takes the instruments through the Earth's radiation belts every three days ("perigee passage") making it necessary to temporarily switch off many of the INTEGRAL instruments including ISGRI. Observation scheduling and public data release is then organised per revolution.

This study is based on all available high-quality public data (at the time the dataset was frozen by us). These are the data from revolutions 21 to 500, i.e. from 15 December 2002 to 18 November 2006. From this time period, we select good data for each of the objects for which we want to derive spectra. The selection criteria are the following:

1. The angular distance between the pointing direction and the position of the object of interest is required to be less than $10^{\circ}$. This is a compromise between maximum exposure time and maximum data quality. For SPI the maximum offaxis angle could be further increased, but for ISGRI, accepting data beyond $10^{\circ}$ radius (which is well into ISGRI's partially coded field of view) reduces the accuracy of the spectra significantly. Since INTEGRAL does mainly pointed observations based on individual proposals (which have emphasised galactic objects so far), the sky exposure achieved by the INTEGRAL instruments up to now is far from uniform. At low galactic latitudes our dataset therefore contains up to a few thousand science windows while only a few hundred are available for the typical object at higher latitudes.

In those cases where a very large exposure is available, we can reduce the selection radius to $8^{\circ}$ in order to improve data quality. Furthermore, if several objects of interest form a closely spaced group, we use a single dataset centred roughly on the centre of the group.

2. Time periods where the data quality was known to be bad such as times near the radiation belt entry or exit of the spacecraft, times shortly after annealings, times during which solar flares strongly raised the background, or times during which hardware problems occurred, were excluded based on the publicly available database of such events included in the OSA software (version 7.0) provided by the INTEGRAL Science Data Centre (ISDC, Courvoisier et al. 2003). This results in the removal of about $31 \%$ of the archival data (based on elapsed time and averaged over the entire mission up to revolution 500).

3. In order to exclude time periods with problems which might have been missed in the compilation of the database used in the previous criterion, quality cuts were imposed 
on a number of "housekeeping" parameters using the SPI housekeeping database compiled at MPE ${ }^{1}$. In particular, the cuts limited the values of the SPI detector temperature, the veto rate of the SPI anti-coincidence shield (ACS), the rate of SPI single interaction events, and the orbital phase. The cuts were derived from inspecting the long term behaviour of these parameters and assuming smoothness of their time dependence. The orbital phase was conservatively limited to the range between 0.1 and 0.87 in order to safely exclude any radiation belt influence. These housekeeping parameter cuts removed another $9.5 \%$ of the archival data (percentage defined as above), mostly at the beginning of the mission.

For detailed analysis of the 20 objects from the B08 catalog we finally utilise the datasets as presented in Table 1.

\section{Data analysis}

\subsection{SPI data analysis using spimodfit}

The spectral data analysis was carried out using the software packages spiselectscw v3.9 and spimodfit v3.0 (both written by Halloin \& Strong 2007) with additional infrastructure software written by DP. The spimodfit package is part of the standard INTEGRAL data analysis software (OSA) since version 7.0.

Using spiselectscw, the data selection criteria described in Sect. 2.1 were applied to the calibrated and pre-binned data from the SPI database at the Max Planck Institute for extraterrestrial Physics. Using spimodfit, a source region model was then fitted to the selected data. The parameters of this model are in our case dynamically constructed by spimodfit from two main components:

1. an isotropic background component;

2. the contributions from those point sources which are within $20^{\circ}$ of the optical axis of the instrument for a given science window.

The isotropic component was modelled as the product of a common scaling factor and a relative sensitivity for each of the 19 detectors of SPI (taking into account the two detector failures during the course of the mission so far). The relative detector sensitivity was permitted to vary on a timescale of 10 revolutions (nearly 30 days) as a compromise between assuming no variability at all (resulting in a degraded fit) and the minimum reasonable variability timescale of one revolution (approx. 3 days, resulting in larger uncertainties due to the increased number of fit parameters). It was verified that assuming a shorter timescale than 30 days did only change the results within their statistical errors.

The common background scaling factor was permitted to vary on a short timescale of $2 \mathrm{~h}$ (corresponding to typically four science windows). This was the conservative choice determined by the actually observed changes in the overall event rate (which is strongly dominated by background) and the aim to minimise the number of model parameters in order to improve sensitivity.

The model components stemming from the point sources are redetermined for every pointing: the algorithm first determines which sources from the input catalog are within $20^{\circ}$ of the pointing axis. Then the tabulated detector response (taking into account the absolute time, spacecraft orientation, the coded mask pattern, and the general spacecraft and instrument mass model)

\footnotetext{
${ }^{1}$ http://www .mpe.mpg.de/gamma/instruments/integral/ spi/www/public_data
}

is used to calculate the relative contribution of each source to the counts in each detector. The model fit parameter is the common scaling factor of the contributions in each detector, i.e. the source flux in the time window corresponding to the pointing. Depending on the variability timescale assumed for the individual source, this flux is then assumed constant for a certain number of pointings.

When the model is constructed, all parameters are tested for relevance, and parameters which do not show an effect on the fit function are excluded. Using a maximum likelihood fit which is initialised from a $\chi^{2}$-fit, the model is then fit to the data and the statistical errors are determined from the covariance matrix. For more details see Halloin \& Strong (2007).

The entire analysis is carried out independently in each energy bin such that boundary conditions such as variability timescales and the input point source catalog can in principle be chosen differently for each energy bin.

In the largest of our datasets (the one containing GRS $1758+258$, see Table 1), the number of degrees of freedom is 74292 per energy bin, while the number of fit parameters is 2582. In the smallest dataset (the one containing NGC 4151) there are only 665 degrees of freedom and 38 fit parameters per energy bin. Thus, for an entire SPI spectrum with 17 energy bins (see also the next section), the total number of degrees of freedom in our datasets is between $\approx 11000$ and $\approx 1.3$ million, the number of fit parameters between 600 and 44000 .

The number of fit parameters is dominated by the background scaling factors and relative detector sensitivities. The source flux for an individual source from the input catalog constitutes only one fit parameter for each energy bin.

Unless otherwise noted, the reduced $\chi^{2}$ values of the model fit in all energy bins for all results presented here were very close to 1.0 .

\subsubsection{Energy binning}

Our default energy binning was chosen a priori based on considerations of SPI energy resolution and flux sensitivity. It consists of 16 energy bins of logarithmically increasing width between 25 and $1000 \mathrm{keV}$. In addition, a narrow energy bin around $511 \mathrm{keV}$ was inserted in order to check for possible annihilation radiation features.

As the analysis showed, many of our sources of interest are too weak to be detected significantly above $200 \mathrm{keV}$ in individual bins of even this coarse (compared to the SPI energy resolution) energy binning. So a second wider binning consisting of 11 bins was introduced for weaker sources. The narrow and the wide binning have the first three bins (up to $48 \mathrm{keV}$ ) in common, then the wider binning roughly combines every two narrow bins up to $500 \mathrm{keV}$ and after that every 3 narrow bins. The bin boundaries of the narrow binning are 25, 31, 39, 48, 60, 75, 93, 116, $144,179,223,278,346,502,520,668,832$, and $1000 \mathrm{keV}$. The bin boundaries of the wider binning are $25,31,39,48,70,103$, $150,219,320,502,520$, and $1000 \mathrm{keV}$.

\subsubsection{Input catalog construction}

As mentioned above, we base our input catalog on the one compiled by B08. This catalog comprises in total 173 sources and states fluxes for them in four energy bands $(25-50 \mathrm{keV}$, 50-100 keV, 100-200 keV, and 200-600 keV). At low energies, all sources of the Bouchet catalog have to be included in the input catalog in order to have a complete model. But as 
Table 1. The 20 objects investigated in this study and the properties of the corresponding datasets.

\begin{tabular}{|c|c|c|c|c|c|c|c|}
\hline Object name $^{a}$ & Object type $^{b}$ & $\begin{array}{c}\text { RA, Dec (FK5) } \\
{\left[^{\circ}\right]}\end{array}$ & galactic $l, b$ & $\begin{array}{c}\text { elapsed } \\
\text { time } \\
{[\mathrm{Ms}]}\end{array}$ & $\begin{array}{c}\text { effective } \\
\text { SPI exposure } \\
{\left[10^{6} \mathrm{~cm}^{2} \mathrm{~s}\right]}\end{array}$ & $\begin{array}{l}\text { var. }^{d} \\
{[\mathrm{ks}]}\end{array}$ & $\begin{array}{c}\text { Data } \\
\text { Set }\end{array}$ \\
\hline Crab & PWN & $083.6332,+22.0145$ & $184.5575,-05.7843$ & 1.33 & 22.7 & - & 1 \\
\hline Vela Pulsar & PWN & $128.8361,-45.1764$ & $263.5520,-02.7873$ & 3.48 & 68.9 & - & 2 \\
\hline NGC 4151 & Seyfert 1.5 & $182.6364,+39.4054$ & $155.0765,+75.0637$ & 0.085 & 5.2 & - & 3 \\
\hline NGC 4945 & Seyfert 2 & $196.3587,-49.4708$ & $305.2681,+13.3374$ & 0.53 & 9.1 & - & 4 \\
\hline Cen A & Radio Gal., Seyf.2 & $201.3651,-43.01911$ & $309.5159,+19.4173$ & 0.56 & 11.5 & - & 5 \\
\hline XTE J1550-564 & $\mathrm{LMXB}, \mathrm{BHC}, \mu \mathrm{Q}$ & $237.7446,-56.4767$ & $325.8822,-01.8271$ & 2.56 & 42.4 & - & 6 \\
\hline 4U 1630-47 & LMXB, BHC & $248.5017,-47.3942$ & $336.9081,+00.2519$ & 3.97 & 154 & - & 7 \\
\hline Swift J1656.3-3302 & Blazar & $254.0690,-33.0359$ & $350.5993+06.3581$ & 10.1 & 151 & - & 10 \\
\hline OAO 1657-415 & HMXB, Pulsar & $255.1996,-41.6731$ & $344.3538,+00.3111$ & 3.97 & 110 & - & 7 \\
\hline GX 339-4 & $\mathrm{LMXB}, \mathrm{BHC}, \mu \mathrm{Q}$ & $255.7062,-48.7897$ & $338.9394,-04.3267$ & 3.36 & 123 & - & 8 \\
\hline 4U 1700-377 & HMXB, NS & $255.9866,-37.8441$ & $347.7544,+02.1735$ & 3.97 & 73.5 & $90,-$ & 7 \\
\hline IGR J17091-3624 & LMXB?, BHC & $257.275,-36.410$ & $349.519,+02.215$ & 7.08 & 214 & - & 9 \\
\hline GX 354-0 & LMXB, NS & $262.9892,-33.8347$ & $354.3022,-00.1501$ & 7.08 & 130 & - & 9 \\
\hline 1E $1740.7-2942$ & $\mathrm{LMXB}$ ?, BHC, $\mu \mathrm{Q}$ & $265.9785,-29.7452$ & $359.1160,-00.1057$ & 7.08 & 134 & - & 9 \\
\hline IGR J17464-3213 & $\mathrm{LMXB} ?, \mathrm{BHC}, \mu \mathrm{Q}$ & $266.5650,-32.2335$ & $357.2552,-01.8330$ & 7.08 & 114 & $90,-$ & 9 \\
\hline GRS 1758-258 & $\mathrm{LMXB}, \mathrm{BHC}, \mu \mathrm{Q}$ & $270.3012,-25.7433$ & $004.5076,-01.3607$ & 10.1 & 403 & - & 10 \\
\hline Ginga 1826-24 & LMXB, NS & $277.3675,-23.7969$ & $009.2724,-06.0878$ & 10.1 & 239 & - & 10 \\
\hline GRS $1915+105$ & $\mathrm{LMXB}, \mathrm{BHC}, \mu \mathrm{Q}$ & $288.7983,+10.9456$ & $045.3656,-00.2194$ & 2.83 & 53.1 & $90,-$ & 11 \\
\hline Cyg X-1 & $\mathrm{HMXB}, \mathrm{BHC}, \mu \mathrm{Q}$ & $299.5903,+35.2016$ & $071.3350,+03.0668$ & 1.94 & 32.9 & 90,180 & 12 \\
\hline Cyg X-3 & $\mathrm{HMXB}, \mathrm{BHC}, \mu \mathrm{Q}$ & $308.1074,+40.9578$ & $079.8455,+00.7001$ & 2.79 & 50.2 & $90,-$ & 13 \\
\hline Control1 $^{e}$ & - & $259.5000,-40.3500$ & $347.7000,-02.1000$ & 7.08 & 181 & - & 9 \\
\hline
\end{tabular}

${ }^{a}$ See Sect. 2.1 for the selection criteria and Sect. 4 for references concerning the source properties.

${ }^{b} \mathrm{PWN}=$ pulsar wind nebula, $\mu \mathrm{Q}=$ microquasar, $\mathrm{NS}=$ binary containing a neutron star, $\mathrm{BHC}=$ containing a black hole candidate, ? = classification uncertain.

${ }^{c}$ Calculated at $30 \mathrm{keV}$ and taking into account the SPI response.

${ }^{d}$ Assumed variability timescale in the analysis at $E<144 \mathrm{keV}$ (first number) and at $E>144 \mathrm{keV}$ (second number); "-" = the source was assumed constant either because it is known to be steady or because the variability timescale and/or amplitude is too small for SPI to detect significant variability.

${ }^{e}$ Control position for the assessment of systematic errors in the analysis of crowded fields (see Sects. 3.3 and 4.21).

the energy increases, many sources in the catalog drop below our flux sensitivity and need no longer be included. In order to optimise the sensitivity of our analysis, we therefore construct three separate catalogs for three energy ranges: (a) 25-144 keV; (b) 144-346 keV; and (c) 346-8000 keV. For range (a), we use the entire Bouchet catalog. For range (b) we use only those sources from the Bouchet catalog which are detected at 100-200 keV (i.e. a flux is stated in B08, not an upper limit) and which have at least $3 \mathrm{mCrab}$ at $50-100 \mathrm{keV}$. Finally, for range (c) we include only those objects from the Bouchet cata$\log$ which have a flux $>6 \mathrm{mCrab}$ at $100-200 \mathrm{keV}$ or which are marked as variable and detected at $50-100 \mathrm{keV}$. Figure 1 shows the positions of the sources in these three input catalogs in galactic coordinates.

The fraction of the sources from the input catalogs which are actually included in the model constructed by spimodfit for an individual dataset, depends on how crowded the field is and how widely spread the pointings are within the search radius. In our datasets (see Table 1) the number of sources included in the model ranges from 1 (in energy range (c) for the least crowded fields) to 83 (in energy range (a) of the most extended among our datasets, the one containing GRS 1758-258).

\subsubsection{Time variability}

The source variability is an important parameter in the flux extraction process. Bright and strongly variable sources can introduce some spurious features in the analysis if their model variability timescale is chosen too long and their true variability timescale and amplitude is large enough for SPI to detect a flux change.

We treat those sources as time-variable which are marked as such in the B08 catalog. These are the following 12 sources (in brackets we give the variability timescale chosen by us based on the ISGRI light curves (Courvoisier et al. 2008) and the sensitivity of SPI): 1A $0535+262(180 \mathrm{ks})$, Vela X-1 (90 ks), GX 301-2 (90 ks), 4U 1700-377 (90 ks), IGR J17464-3213 (90 ks), Sco X-1 (90 ks), Swift J1753.5-0127 (2.6 Ms), Aql X-1 (180 ks), GRS 1915+105 (90 ks), Cyg X-1 (90 ks, $180 \mathrm{ks}$ for $E>144 \mathrm{keV}$ ), EXO 0331 (2.6 Ms), Cyg X-3 (90 ks). Cyg X-1 is the only source which is significantly variable over the entire energy range. All other variable sources are only treated as signifcantly variable (given the SPI sensitivity) up to $144 \mathrm{keV}$.

The minimum timescales used here were determined from a study of model fit convergence. We found that our model fits do not converge well if we assume timescales much below $90 \mathrm{ks}$ resulting in increased statistical errors on all fit parameters. Particularly in the case of the bright and strongly variable source 4 U 1700-377, B08 used a variability timescale of $\approx 3 \mathrm{ks}$ (the minimum possible due to the way the data is stored). For such sources, dedicated studies are necessary to further increase the accuracy of spectra for short integration times. However, it is our aim here to provide long-term average spectra with a coherent treatment of all sources.

For the variable sources, the analysis yields a separate spectrum for each variability time bin, or in other words a separate light curve for each energy bin. We derive the average 

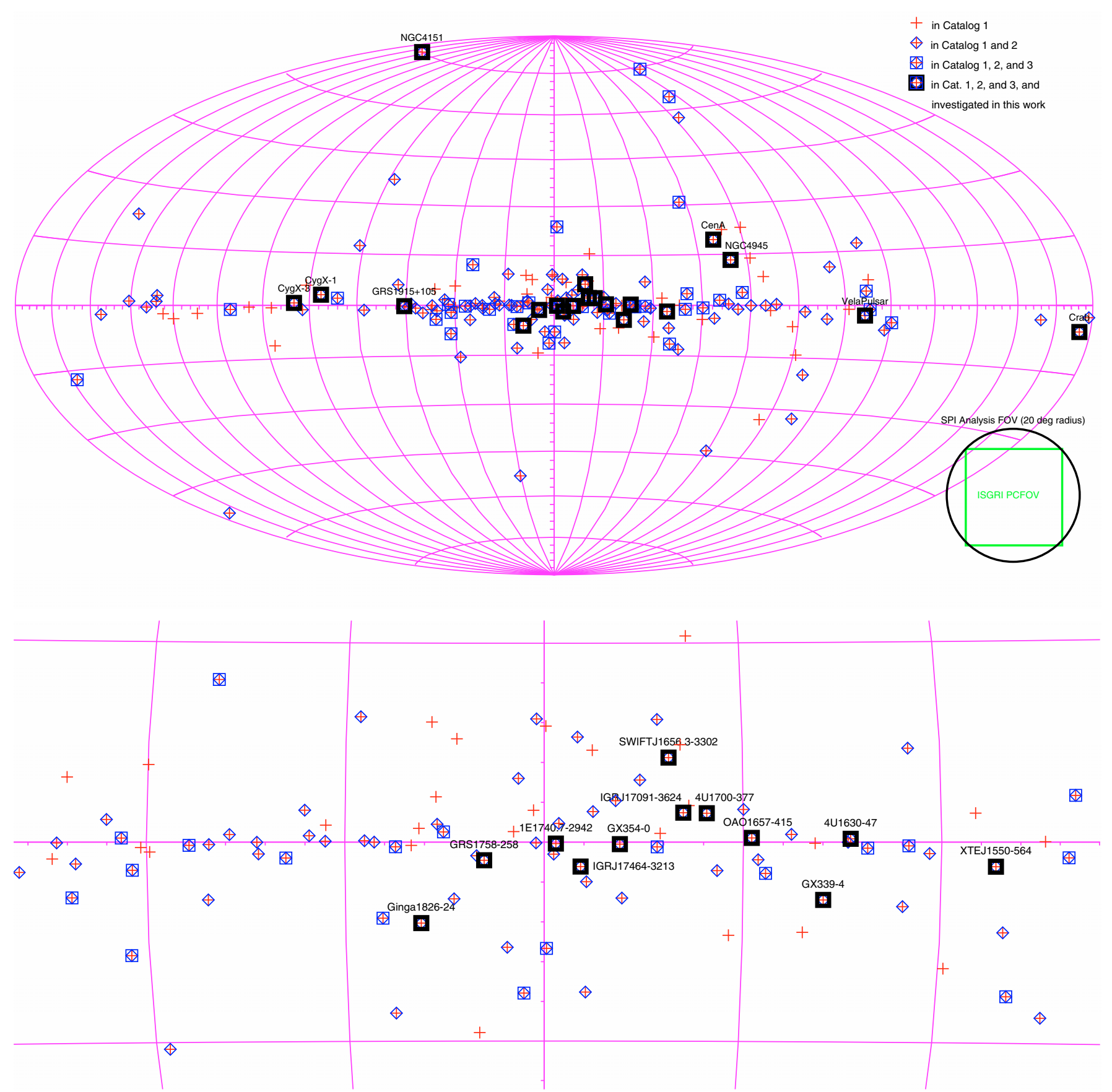

Fig. 1. a) (Top) the positions of the sources from the input catalogs used in this study for the analysis of the SPI data in three separate energy ranges: catalog 1 (25-144 keV, crosses, 173 objects, i.e. the entire B08 catalog), catalog 2 (144-346 keV, diamonds, 129 objects), and catalog 3 $(346-1000 \mathrm{keV}$, squares, 52 objects) on an all-sky map in galactic coordinates. The 20 objects investigated in this work are marked as thick squares. The grid mesh size $15^{\circ} \times 15^{\circ}$. Also shown are the extensions of the SPI analysis field of view and the ISGRI partially coded field of view. b) (Bottom) the enlarged galactic centre region of a). The catalogs for higher energies are derived by successively removing weak sources from the initial catalog, i.e. a lower energy catalog contains all higher-energy ones. See Sect. 3.1.2.

spectrum by applying a weighted least-squares procedure described in Alvarez-Gaumé et al. (2004), page 14, to the light curves from each energy bin.

\subsubsection{Postprocessing and spectral model fitting}

The analysis is sped up by processing each energy bin on a separate processor reducing the required computing time by about a factor equal to the number of energy bins, i.e. from several days to a few hours for each source data set.

In a final step, the data from each processor are collected, where necessary the time-averaging of the spectra is performed, and the spectra are converted to the PHA format suitable for the spectral analysis with XSpec 12.3.1 (Arnaud et al. 2007) which is then used for the fitting of spectral models taking into account the SPI response and energy-redistribution. 


\subsection{ISGRI data analysis}

The analysis of the INTEGRAL IBIS/ISGRI data is based on a cross-correlation procedure between the recorded image on the detector plane and a decoding array derived from the mask pattern (see Goldwurm et al. 2003). Standard spectral extraction has been applied as provided by the OSA 7.0 software package, using the same energy binning and science windows as for the SPI data.

\subsection{Statistical and systematic errors}

The statistical errors of the photon count rates in each energy bin are determined both for the ISGRI and the SPI data by the fit software taking into account proper Poisson count statistics both for background and source signal.

In addition there is a systematic error on the flux calculation stemming from the uncertainty of the calibration due to the limited amount of calibration data and the propagation of other uncertainties of the calibration process. We derive the relative error of the calibration of the spectra by assuming a shape for the Crab Nebula spectrum and independence of energy, i.e. that the uncertainty caused by the error on the calibration is the same percentage of the measured count rate for each energy bin. This constant percentage is determined by varying it until the reduced $\chi^{2}$ from the fit of the assumed spectral shape to the Crab spectrum is close to 1.0 for the complete energy range.

For both the ISGRI and the SPI data, we follow the INTEGRAL Cross-calibration status document by Jourdain et al. (2008) and assume the Crab Nebula spectrum between $25 \mathrm{keV}$ and $1 \mathrm{MeV}$ to be a broken powerlaw with break energy fixed at $100 \mathrm{keV}$ (which is close to the break energy we actually measure, see Sect. 4.1). With this assumption we derive a calibration error of our ISGRI and SPI spectra of $0.5 \%$ in each energy bin. Unless otherwise noted, this error has been added in all spectral plots shown in this paper. For the fainter sources, it is, however, negligible.

The Crab is a bright source in a relatively quiet field without many other neighbouring hard X-ray sources. In order to assess the possible systematic errors arising in the analysis of crowded fields in the galactic bulge where some of our 20 sources of interest are located, we have added a control position to our input catalog at $\mathrm{RA}=259.5^{\circ}, \operatorname{Dec}=-40.35^{\circ}\left(l=347.7^{\circ}, b=\right.$ $\left.-2.1^{\circ}\right)$. This position was randomly chosen in an empty region (with respect to the B08 catalog) of the crowded field near GX 354-0. The analysis for this artificial reference source is discussed in Sect. 4.21.

\section{Results}

In this section we present our spectral analysis results and discuss the agreement of our SPI and ISGRI spectra with each other and with previously published measurements for each object in detail. The numerical results are later summarised in Tables 2, 3 and 5 in Sect. 5 where we also discuss the agreement with the fluxes given by B08.

Studying data below $25 \mathrm{keV}$ would exceed the scope of this paper. Also, due to the small field-of-view of the INTEGRAL soft X-ray instrument Jem-X, a soft-X-ray dataset truly concurrent with our SPI and ISGRI dataset would have meant shrinking the SPI and ISGRI exposure by more than a factor two. When we compare to previously published measurements, this lack of soft X-ray data leads often to poorly constrained low energy features such as cutoffs near our lower energy limit ( $25 \mathrm{keV})$ or soft photon temperatures in thermal Comptonisation models. Where necessary, we try to overcome this by taking the appropriate values from the literature.

In the following, unless otherwise noted, the normalisation parameters "Norm" or "Norm" 1 " in the spectral fit results are given in units of photons $\mathrm{keV}^{-1} \mathrm{~cm}^{-2} \mathrm{~s}^{-1}$. They correspond to the flux at $1 \mathrm{keV}$. The letter $\Gamma$ designates a powerlaw spectral index. If we need to introduce a second power-law component for high-energy emission, we use the XSpec model PEGPWRLW with peg energy $200 \mathrm{keV}$. The normalization of it ("Norm 2 ") is given as the differential flux at $200 \mathrm{keV}$.

In the figures in this section we show the spectra unfolded by XSpec with statistical errors (slightly increased by the calibration errors discussed in Sect. 3.3). The solid line always indicates the fitted model (as a step function according to the energy binning). If the model has several components, these are also separately shown with dashed lines.

\subsection{Crab Nebula}

The Crab Nebula is the standard candle of high-energy astronomy. Because of the steady, strong emission from this pulsar wind nebula, it has always been among the first objects to be observed by new instruments. For a recent compilation of X-ray data on the Crab see, e.g., Kirsch et al. (2005). Still, its high energy spectrum is not too well known. Many instruments use an assumption about the Crab's spectral shape as the basis of their calibration and can hence not say much about the fine details of the true spectrum. This is also the case for INTEGRAL/ISGRI. On the other hand, INTEGRAL/SPI with its high energy resolution could measure a Crab spectrum which is less dependent on such assumptions about the spectral shape.

Figure 2a shows the result of our analysis of the SPI Crab spectrum. A good fit is a broken powerlaw (XSpec model "bknpow") with the following parameters:

$\Gamma_{1}=2.11 \pm 0.01$

$E_{\text {break }}=(81 \pm 11) \mathrm{keV}$

$\Gamma_{2}=2.20 \pm 0.01$

Norm $=11.6 \pm 0.5$

with a reduced $\chi^{2}$ of 0.8 for 13 degrees of freedom (see also Sect. 3.3).

The corresponding ISGRI dataset gives the following Crab spectrum (Fig. 2b): $\Gamma_{1}=2.119 \pm 0.006, E_{\text {break }}=(96 \pm 4) \mathrm{keV}$, $\Gamma_{2}=2.36 \pm 0.02$, Norm $=10.7 \pm 0.3$, with a reduced $\chi^{2}$ of 1.3 for 11 d.o.f. (see Sect. 3.3).

This needs to be compared to our best fit for the SPI data. The shape is in very good agreement below $100 \mathrm{keV}$. Table 4 (see Sect. 5) gives our measurements of the Crab flux in several energy bands. The model fluxes between 25 and $100 \mathrm{keV}$ $\left(0.2287 \pm 0.0004 \mathrm{~cm}^{-2} \mathrm{~s}^{-1}\right.$ for SPI and $0.2046 \pm 0.0004 \mathrm{~cm}^{-2} \mathrm{~s}^{-1}$ for ISGRI) differ by a factor $1.11 \pm 0.01$ (SPI/ISGRI) which has to be regarded as a measurement of the systematic difference in absolute flux normalisation.

Above the fixed break, the ISGRI spectrum is significantly softer than the SPI spectrum. However, as the ISGRI response was made (by the authors of the OSA 7 ISGRI response) to give the Crab spectrum a powerlaw above $100 \mathrm{keV}$, this deviation tells us that there are probably significant systematic errors in the ISGRI calibration at the upper end of its energy range in addition to the calibration errors derived by us in Sect. 3.3.

Our SPI spectrum is in very good agreement with that derived from SPI data up to $1 \mathrm{MeV}$ by Jourdain et al. (2008) whose 

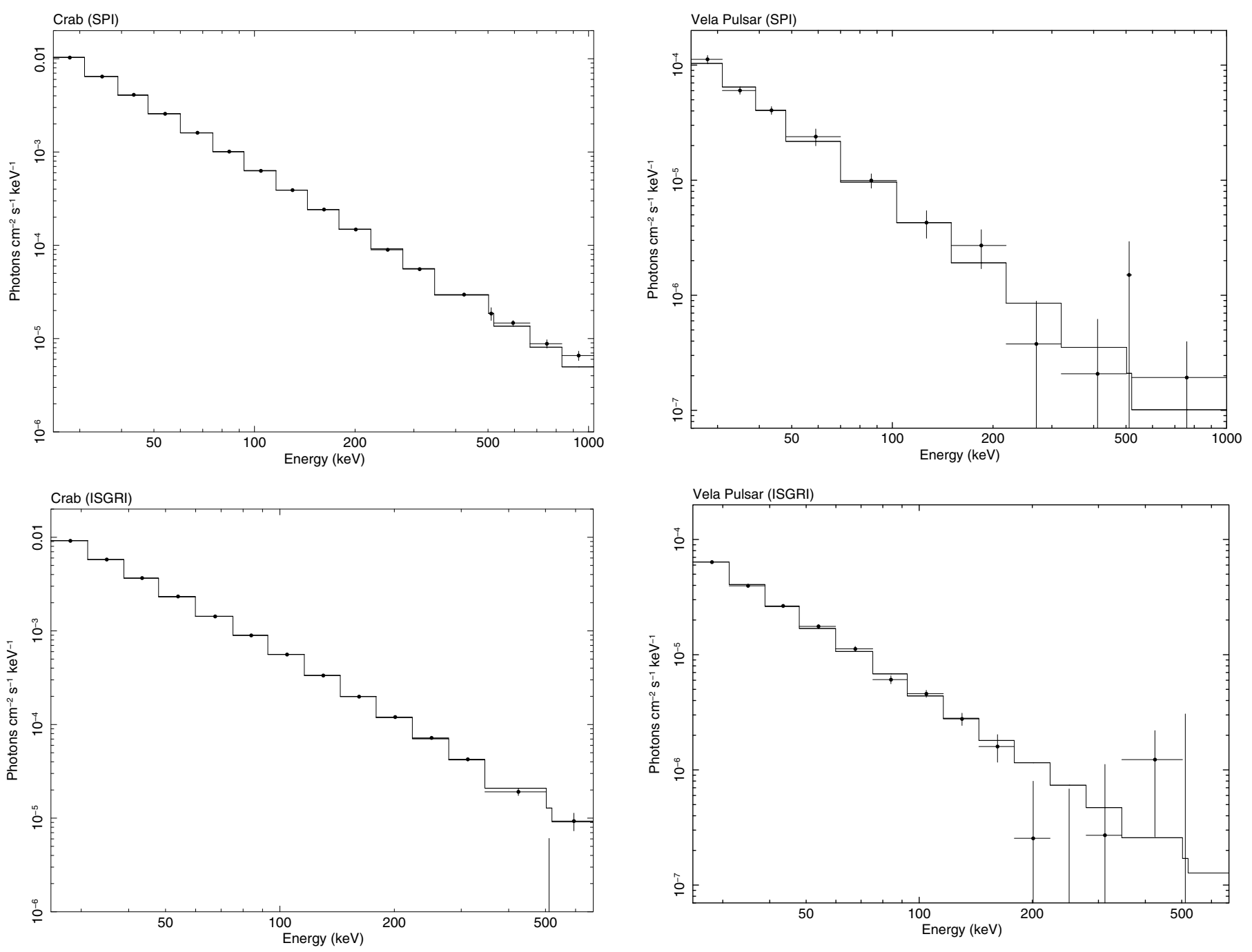

Fig. 2. The unfolded spectra of the Crab Nebula as derived from dataset 1 (see Table 1) with statistical errors: a) (top) SPI spectrum b) (bottom) ISGRI spectrum; fit function is in both cases a broken powerlaw. Parameters of both fits are given in Sect. 4.1.

analysis was not based on spimodfit. Furthermore, the smooth fit of the narrow energy bin around the $511 \mathrm{keV}$ line, which is also a strong background line of the instrument, demonstrates that the background determination works well.

\subsection{Vela pulsar}

The other pulsar wind nebula/pulsar in our set of sources, the Vela PWN, is generally regarded as similar to the Crab. However, in hard X-rays the object is about two orders of magnitude fainter even though it is nearly an order of magnitude closer. It has been studied in detail by all high-resolution soft X-ray observatories (see e.g. Mangano et al. 2006, and references therein).

Figure 3a shows the spectrum from our analysis of the SPI Vela Pulsar data. Because of the weakness of the source, we choose the wider energy binning in the SPI analysis. A simple powerlaw with $\Gamma=2.13 \pm 0.10$, Norm $=0.12 \pm 0.05$ describes the spectrum well. The reduced $\chi^{2}$ of the fit is 0.55 for 9 d.o.f.

The SPI spectrum is in good agreement with our corresponding ISGRI spectrum (Fig. 3b) which has the parameters

Fig. 3. The unfolded spectra of the Vela Pulsar and Pulsar Wind Nebula derived from dataset 2 (see Table 1) with statistical errors: a) (top) SPI spectrum; (b) (bottom) ISGRI spectrum. Fit function is in both cases a simple powerlaw (parameters are given in Sect. 4.2).

$\Gamma=2.04 \pm 0.04$, Norm $=(0.057 \pm 0.008)$, with a reduced $\chi^{2}$ of 1.4 for 13 d.o.f.

The hard X-ray spectrum of this source was studied up to $200 \mathrm{keV}$ by Mangano et al. (2006) using Beppo-SAX data. They find a spectral index of $2.00 \pm 0.05$ at $15-200 \mathrm{keV}$ which is in very good agreement with our measurements.

At energies above $200 \mathrm{keV}$, our analysis does not show significant evidence for emission. On the other hand, our measurement is well consistent with a continuation of the spectrum in a way similar to what we observe for the Crab. Given the nature of the source and its morphological similarity to the Crab, this is to be expected. OSSE detected the Vela PWN only marginally between 200 and $760 \mathrm{keV}$ with a spectral index of $1.8 \pm 0.3$ (Strickman et al. 1996b) compatible with a continuation of our spectrum without a break.

\subsection{NGC 4151}

The prototypical Seyfert galaxy NGC 4151 is one of the best studied active galaxies. For a comprehensive review on this object see Ulrich (2000). Unfortunately, this is our smallest dataset with less than $100 \mathrm{ks}$ observation time. We show the 

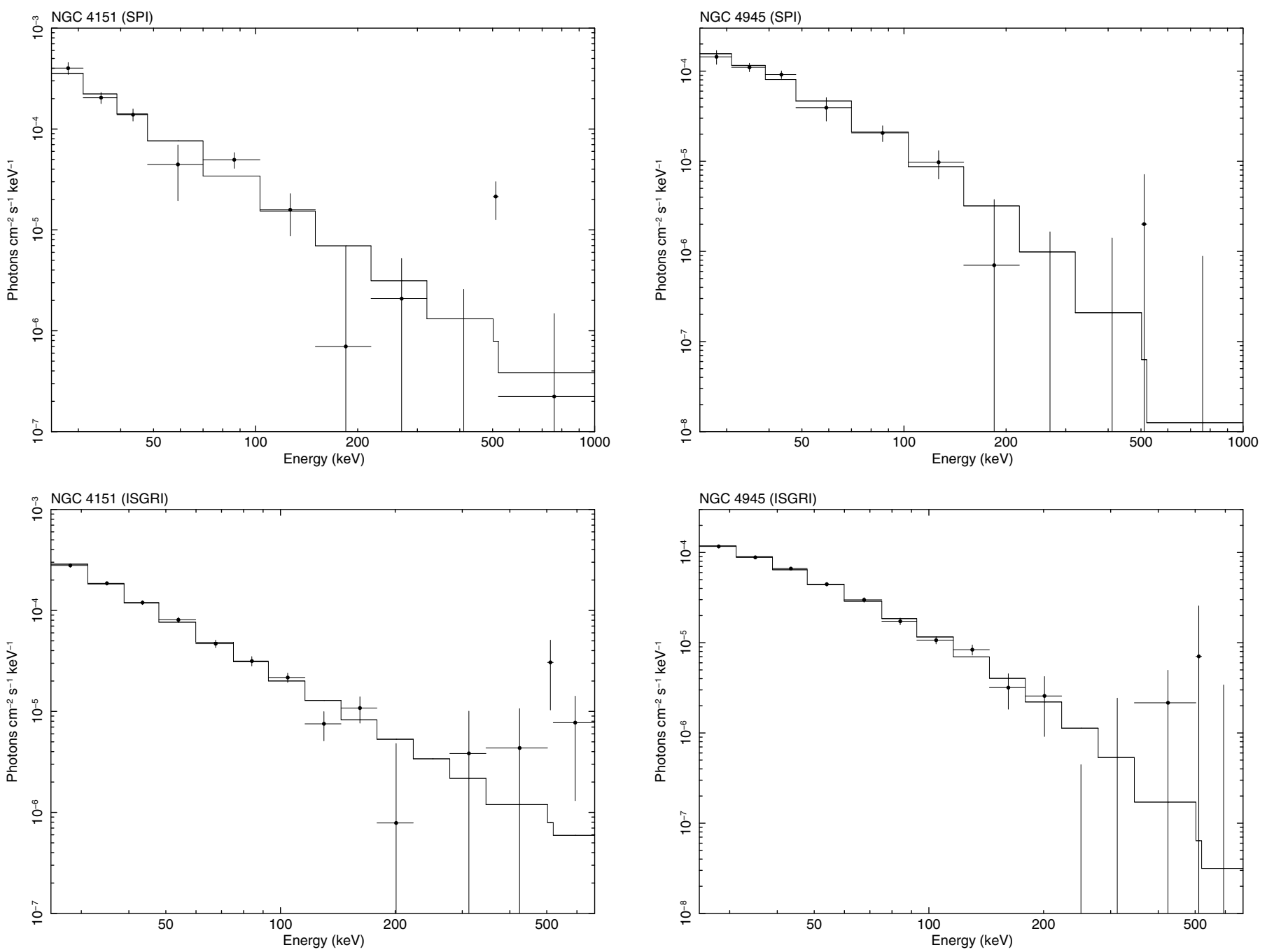

Fig. 4. The unfolded spectra of NGC 4151 derived from dataset 3 (which has very low exposure, see Table 1) with statistical errors: a) (top) SPI spectrum; b) (bottom) ISGRI spectrum. The fit function is in both cases a powerlaw (parameters are given in Sect. 4.3).

resulting spectra here only for completeness. A detailed study of this source using INTEGRAL data up to $300 \mathrm{keV}$ was published by Beckmann et al. (2005) (they use only ISGRI data and get a larger dataset by using data taken during SPI annealings).

Figure $4 \mathrm{a}$ shows the SPI spectrum. The best fit is a simple powerlaw with $\Gamma=2.10 \pm 0.17$, Norm $=0.38 \pm 0.25$, and a reduced $\chi^{2}$ of 1.4 for 9 d.o.f. The excess in the annihilation line bin is marginal ( $2.4 \sigma$ significance).

The ISGRI spectrum (Fig. 4b) gives a compatible fit result with $\Gamma=2.03 \pm 0.06$, Norm $=0.24 \pm 0.05$, reduced $\chi^{2}=1.4$ for 13 d.o.f.

Fitting a powerlaw with exponential cutoff to the ISGRI data as in Beckmann et al. (2005) improves the fit slightly $\left(\chi^{2}=1.3\right)$ and results in values well compatible with previously published measurements: $\Gamma=1.57 \pm 0.24, E_{\text {cutoff }}=121 \pm$ $55 \mathrm{keV}$, Norm $=0.07 \pm 0.04$.

\subsection{NGC 4945}

NGC 4945 is the brightest Seyfert 2 and the second brightest hard X-ray source (after NGC 4151) of all radio-quiet AGN (Done et al. 1996). Its X-ray spectrum has been extensively studied by various authors (see e.g. Itoh et al. (2008) and

Fig. 5. The unfolded spectra of NGC 4945 derived from dataset 4 (which has low exposure, see Table 1) with statistical errors: a) (top) SPI spectrum; (b) (bottom) ISGRI spectrum. The fit function is in both cases a powerlaw with exponential cutoff and fixed photo-electric absorption (parameters are given in Sect. 4.4).

references therein). As a Seyfert 2, the source is strongly absorbed at lower energies. Itoh et al. (2008) using Suzaku measure a hydrogen column density $N_{\mathrm{H}}$ of $5.4 \times 10^{24} \mathrm{~cm}^{-2}$ and a hard X-ray spectrum with a powerlaw index of $1.5 \pm_{0.1}^{0.2}$ and an exponential cutoff at an energy of $150 \pm_{50}^{100} \mathrm{keV}$ (in agreement with previous measurements).

Our dataset for NGC 4945, like the one for NGC 4151, has relatively low exposure. The hydrogen column density for this source is by far the highest of all the sources in our sample. We find that it is still low enough to be negligible for our SPI spectral modelling but the ISGRI sensitivity below $50 \mathrm{keV}$ is high enough to make the spectral fits sensitive to the presence of the absorption. In order to compare better with previous measurements, we therefore include the photoelectric absorption in the spectral model (Xspec model WABS) and fit an absorbed powerlaw with exponential cutoff to the SPI and the ISGRI spectrum fixing the hydrogen column and the cutoff energy to the values found by Itoh et al. (2008).

Figure 5a shows the resulting SPI spectrum. The best fit parameters are $\Gamma=1.66 \pm 0.17$, Norm $=0.061 \pm 0.039$ with a reduced $\chi^{2}$ of 0.4 for 9 d.o.f. 

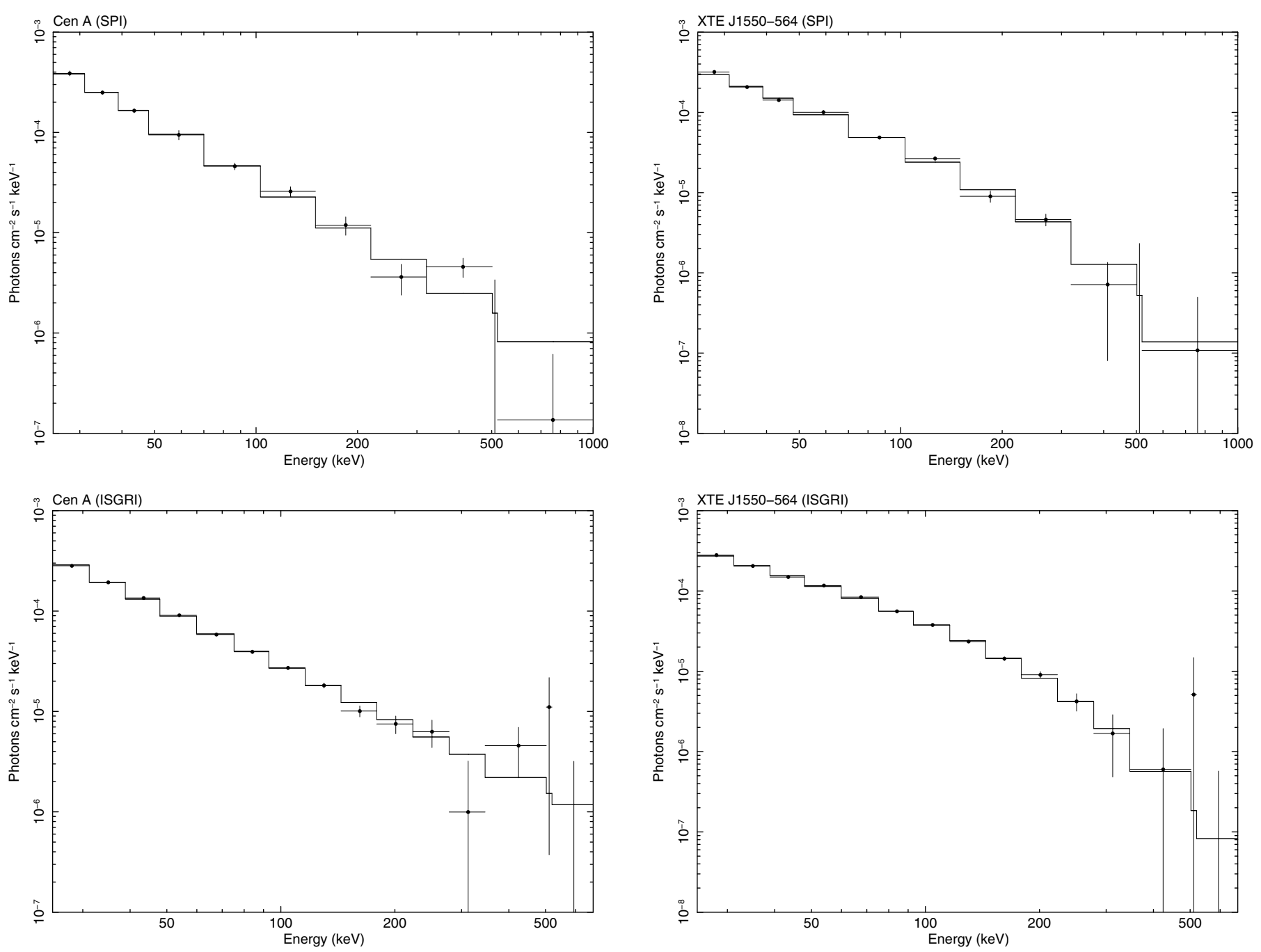

Fig. 6. The unfolded spectra of Cen A derived from dataset 5 (see Table 1) with statistical errors: a) (top) SPI spectrum; b) (bottom) ISGRI spectrum. The fit function is in both cases a powerlaw (parameters are given in Sect. 4.5).

The ISGRI spectrum is shown in Fig. 5b. Keeping $N_{\mathrm{H}}$ fixed as for SPI, the best fit parameters are $\Gamma=1.56 \pm 0.05$, Norm $=$ $0.033 \pm 0.006$ with a reduced $\chi^{2}$ of 0.6 for 13 d.o.f.

SPI and ISGRI spectrum are both consistent with each other and with the spectra measured by Itoh et al. (2008).

\subsection{Cen $A$}

The nearby radio galaxy Cen $\mathrm{A}$ has been observed by all X-ray observatories (see Rothschild et al. 2006, and references therein). It has been classified as a Seyfert 2 (see Dermer \& Gehrels 1995). The consistently observed hard X-ray spectrum is, in spite of flux variability, a simple powerlaw of index $\approx 1.8$ (Rothschild et al. 2006) which extends to the $\mathrm{MeV}$ range where the spectrum steepens (Steinle et al. 1998).

Even though the available SPI exposure for Cen A is modest, we obtain a clear detection also beyond $200 \mathrm{keV}$. The SPI spectrum (Fig. 6a) gives the best fit parameters $\Gamma=1.89 \pm 0.05$, Norm $=0.20 \pm 0.04$, and a reduced $\chi^{2}$ of 1.2 for 9 d.o.f..

Similarly, the ISGRI spectrum (Fig. 6b) gives a best fit with $\Gamma=1.80 \pm 0.02$, Norm $=0.117 \pm 0.009$ and a reduced $\chi^{2}$ of 0.9 for 13 d.o.f.

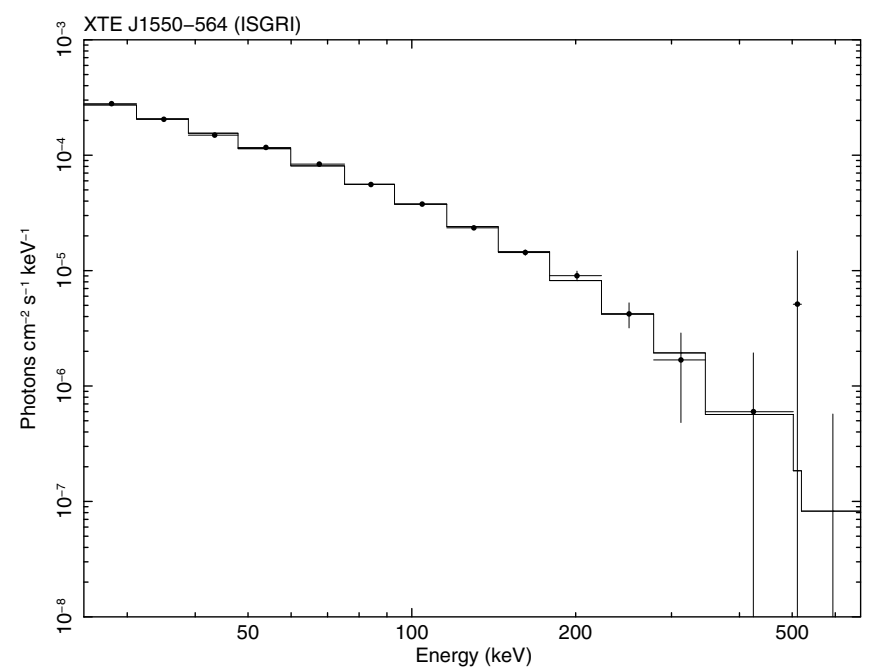

Fig. 7. The unfolded spectra of XTE J1550-564 derived from dataset 6 (see Table 1) with statistical errors: a) (top) SPI spectrum; b) (bottom) ISGRI spectrum (increased errors were required, see text). The fit function is in both cases a powerlaw with exponential cutoff (parameters are given in Sect. 4.6).

SPI and ISGRI spectral shape are in excellent agreement with each other and previous measurements.

\subsection{XTE J1550-564}

The low-mass X-ray Binary XTE J1550-564 has been classified as a microquasar and a black hole candidate. Since its discovery in 1998 (Smith 1998) it has been subject of several detailed studies and was found to be similar to Cyg X-1, however, with lower luminosity (see e.g. Wu et al. 2007, and references therein). Sturner \& Shrader (2005) studied the source using RXTE and INTEGRAL data and found that it stayed in its lowhard spectral state even when going through modest outbursts. According to the ISGRI lightcurve of the source (Courvoisier et al. 2008), XTE J1550-564 had only two short major outbursts with INTEGRAL coverage and was otherwise in a low state. The time-averaged spectrum we are determining here should therefore be close to the low-hard type, i.e. consistent with thermal Comptonisation which is essentially a powerlaw with exponential cutoff in our energy range. 


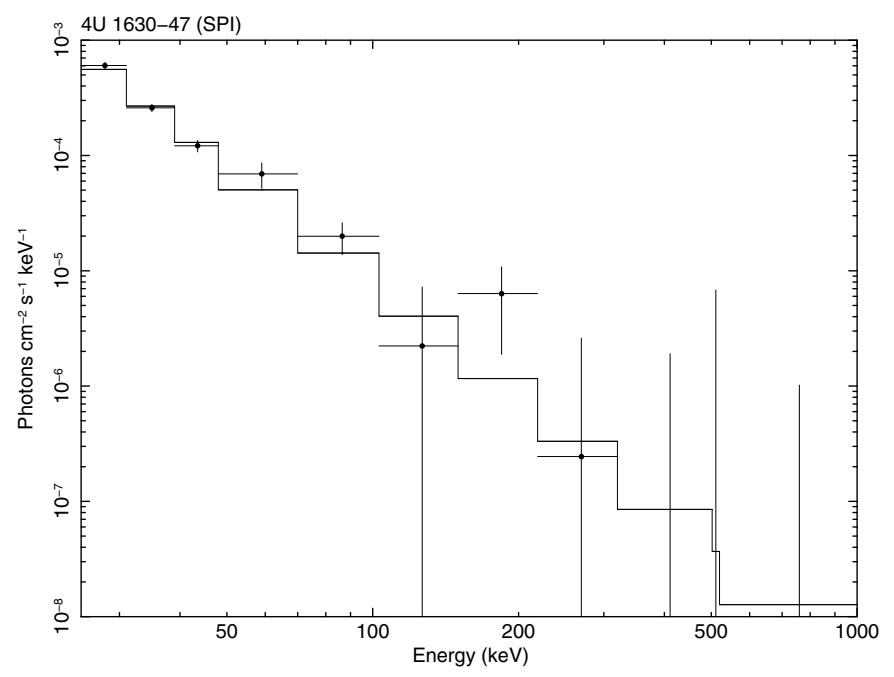

Fig. 8. The unfolded spectrum of $4 \mathrm{U} 1630-47$ derived from the SPI dataset 7 (see Table 1) with statistical errors. The corresponding ISGRI data is flawed because of source contamination by IGR J16358472 (see text). The fit function is a powerlaw (parameters are given in Sect. 4.7).

We find that a powerlaw with exponential cutoff fits the SPI data reasonably. From the SPI spectrum (Fig. 7a) we get the parameters $\Gamma=1.36 \pm 0.09, E_{\text {cutoff }}=(206 \pm 50) \mathrm{keV}$, Norm $=$ $0.031 \pm 0.010$ with a reduced $\chi^{2}$ of 1.6 for 8 d.o.f.

For the ISGRI spectrum we don't achieve a reduced $\chi^{2}$ below 2.0 with any common model (we tried cutoff powerlaw, thermal Comptonisation, and black body + powerlaw) although the residuals don't show any clear trend in the deviations. This can be explained by additional systematic errors in the image reconstruction caused by the complex source region. Allowing for this by doubling the assumed calibration errors from $0.5 \%$ to $1.0 \%$ (see also Sturner \& Shrader 2005), we get a reduced $\chi^{2}$ of 1.3 for 12 d.o.f. The best fit parameters are marginally consistent with the ones obtained from the SPI data: $\Gamma=1.00 \pm 0.05$, $E_{\text {cutoff }}=(108 \pm 9) \mathrm{keV}$, Norm $=0.009 \pm 0.002$. This is due to higher photon fluxes measured by ISGRI below $200 \mathrm{keV}$. Above $200 \mathrm{keV}$, the fluxes are consistent (cf. Table 5).

For comparison with earlier publications, we also fit the thermal Comptonisation model (COMPTT) by Titarchuk (1994) which is the best fit to the ISGRI and SPI data in Sturner \& Shrader (2005). Averaging over their datasets, they obtain a plasma temperature $k T=(48.4 \pm 2.7) \mathrm{keV}$, a plasma optical depth $\tau_{\mathrm{p}}=1.48 \pm 0.07$, and a soft photon temperature $k T_{0}=$ $(0.5 \pm 0.14) \mathrm{keV}$. Since we lack the data between $3 \mathrm{keV}$ and $25 \mathrm{keV}, k T_{0}$ is not well constrained. We therefore fix it to the value $0.5 \mathrm{keV}$ by Sturner \& Shrader. From the SPI data we then obtain $k T=(56.9 \pm 7.7) \mathrm{keV}$ and $\tau_{\mathrm{p}}=1.32 \pm 0.18\left(\right.$ reduced $\chi^{2}=$ 1.3 for 9 d.o.f.) in good agreement with Sturner \& Shrader.

\section{7. $4 U 1630-47$}

The X-ray transient 4U 1630-47 has been classified as a LMXB, a very likely black hole candidate, and has also been found to show indications of the presence of jets but is not yet regarded as a microquasar (see Kubota et al. 2007, and references therein). During the observation time covered by our dataset, the source showed frequent but short outbursts. The time-averaged spectrum should therefore be a superposition of the various states the source can be in, with the low state dominating. Tomsick et al. (2005) analyse RXTE and INTEGRAL data from 4U 1630-47. They distinguish five different spectral states which they all fit

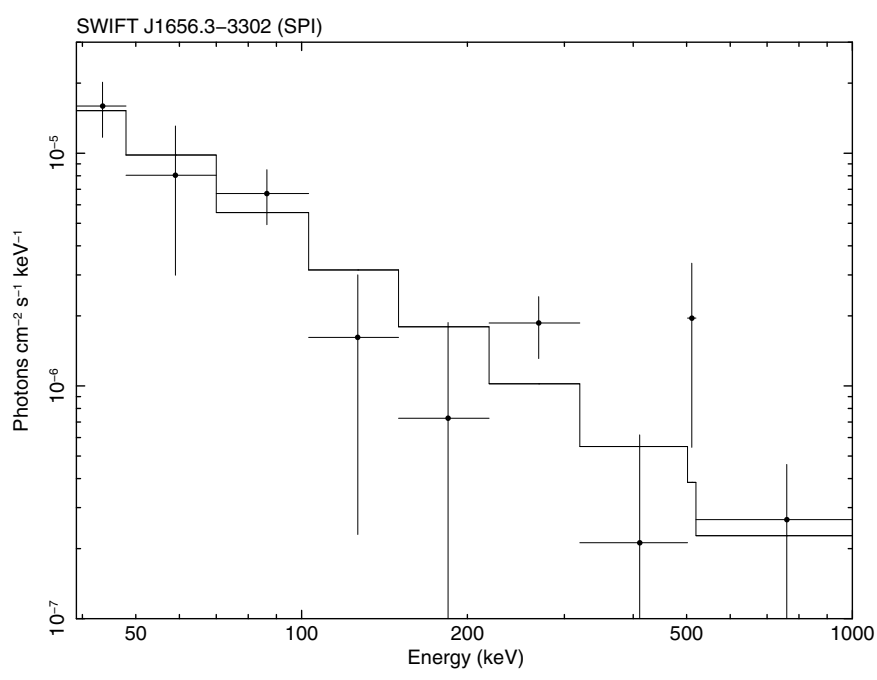

Fig. 9. The unfolded SPI spectrum of Swift J1656.3-3302 derived from dataset 10 (see Table 1) with statistical errors. The fit function is a powerlaw (parameters are given in Sect. 4.8).

with a two-component model made from a black body with interstellar absorption and a powerlaw. They find that the parameters of this model vary strongly between the states. The lowest flux state is found to be the one where the emission is dominated by black body emission $(k T \approx 1.4 \mathrm{keV})$ below $15 \mathrm{keV}$, and above that by a steep powerlaw with index $\Gamma \approx 3$. A simple powerlaw is also the best fit to our spectra of this on average relatively weak source.

The SPI spectrum for $4 \mathrm{U} 1630-47$ (Fig. 8) is best fit by a powerlaw with $\Gamma=3.3 \pm 0.2$ and Norm $=33 \pm 21$. The reduced $\chi^{2}$ is 0.6 for 9 d.o.f.

The ISGRI spectrum may suffer from source contamination from the neighbouring source IGR J16358-4726, especially at higher energies (see also Tomsick et al. 2005). We try to account for this approximately by increasing the systematic errors to $5 \%$. Fitting a powerlaw then results in $\Gamma=2.33 \pm 0.24$, Norm $=0.04 \pm 0.04$ with a reduced $\chi^{2}=1.0$ for 13 d.o.f. which is much harder than the SPI spectrum.

Furthermore, the observed fluxes (see Table 5) from SPI and ISGRI differ strongly at low energies while the SPI measurements agree with those from B08 who also note that the source may be confused with IGR J16358-472.

We conclude that the neighbouring source IGR J16358-472 may be hampering the measurement for ISGRI or SPI or both. Since this distortion is strongly dependent on the instrument characteristics, the effects are different for SPI and ISGRI and lead very probably to the observed difference in the spectra. The smaller differences between our spectra and those from Bouchet are to be expected since here the same instrument is used. The fact that the SPI spectrum agrees well with RXTE measurements of 4U 1630-47 in a low state by Tomsick et al. (2005) argues for the better quality of the SPI spectrum. Also, our source model for the SPI data explicitely includes IGR J16358-472. The SPI analysis should therefore in this case be more immune to contamination than the ISGRI analysis.

\subsection{Swift J1656.3-3302}

The recently discovered source Swift J1656.3-3302 (Okajima et al. 2006) has been identified with a high redshift $(z=2.4)$ blazar (Masetti et al. 2008). The late discovery of this source is due to its hard spectrum, i.e. its weakness at soft X-rays, and to its location near the galactic bulge which makes for 
a very complex field of view with many variable and bright neighbouring sources. Probably because many bright sources are very near the edge of the partially coded field of view when Swift J1656.3-3302 is near its centre, our standard modelling technique turned out to be inadequate for extracting a SPI spectrum of Swift J1656.3-3302 from a dataset centred at the source. The quality of the spimodfit fits was never acceptable in more than a few energy bins. But when the source was observed by SPI with high exposure and off-axis by at least $5^{\circ}$ in the dataset selected for GRS 1758-258 (dataset 10, see Table 1), the extraction of a useful spectrum with our standard input catalog and variability information was possible in all energy bins except for the lowest two. The corresponding ISGRI spectrum of Swift J1656.3-3302 from dataset 10, however, was not useful since the object was too far outside the ISGRI fully coded field of view. We therefore present in Fig. 9 only the SPI spectrum for this source. It is well described by a simple powerlaw with $\Gamma=$ $1.49 \pm 0.18$ and Norm $=0.004 \pm 0.005$. The reduced $\chi^{2}$ is 1.0 for 7 d.o.f.

An ISGRI spectrum for Swift J1656.3-3302 was measured by Masetti et al. (2008). They find a spectral index of $1.64 \pm$ 0.16 in agreement with our measurement.

\subsection{OAO 1657-415}

The high mass X-ray binary OAO $1657-415$ is a system consisting of an eclipsing $38 \mathrm{~s}$ pulsar and a highly reddened B supergiant with orbital period 10.4 d (see e.g. Audley et al. 2006, and references therein). The object has been studied since its discovery in the 1970 s by a fair number of authors with several X-ray observatories up to about $160 \mathrm{keV}$, recently also with INTEGRAL (Filippova et al. 2005; Barnstedt et al. 2008). In the range from 3 to $160 \mathrm{keV}$ they find consistently an absorbed, exceptionally hard spectrum with index $\Gamma \approx 1.0$ which then softens with an exponential cutoff at energies around $25 \mathrm{keV}$.

We fit our time-averaged (and therefore also phase-averaged) SPI spectrum initially with the simplest of these models, the powerlaw with exponential cutoff. We obtain $\Gamma=1.17 \pm 0.23$ and $E_{\text {cutoff }}=(23.9 \pm 3.2) \mathrm{keV}$ with a reduced $\chi^{2}$ of 1.4 for 8 d.o.f., in good agreement with previous measurements.

Inspecting the residuals, we find marginal evidence for a hard tail diverging from the model above $150 \mathrm{keV}$. The feature is not excluded by previous studies since they did not cover this energy range. The significance of the hard tail is about $3 \sigma$ which does not warrant a much more detailed study. In order to quantify the possible flux of this feature, we add a powerlaw to the model keeping $E_{\text {cutoff }}$ fixed. This results in a lower reduced $\chi^{2}$ of 0.7 and best fit parameters $\Gamma_{1}=1.23 \pm 0.07$, Norm $_{1}=0.16 \pm 0.04$, $\Gamma_{2}=1.27 \pm 0.58$ and a flux of the second component at $200 \mathrm{keV}$ of $(2.1 \pm 1.0) \times 10^{-6} \mathrm{~cm}^{-2} \mathrm{~s}^{-1} \mathrm{keV}^{-1}$. Figure 10a shows the fit result with the added powerlaw.

For the ISGRI data, we increase the statistical errors by $5 \%$ of the flux in order to get take into account the larger systematic uncertainties of the analysis (this is the same dataset as the one for 4U 1630-47 where this increase was also necessary).

If we fit the same model with added powerlaw as for the SPI data, we get reduced $\chi^{2}$ of 0.4 for 10 d.o.f. compatible with the SPI fit. The parameters of the second powerlaw are essentially unconstrained. The reality of the potential hard tail in the SPI data is not ruled out.

If we fit with the powerlaw with exponential cutoff without the second powerlaw, we get a reduced $\chi^{2}$ of 0.4 for 12 d.o.f. and best fit parameters $\Gamma_{1}=1.4 \pm 0.3, E_{\text {cutoff }}=25 \pm 4 \mathrm{keV}$,
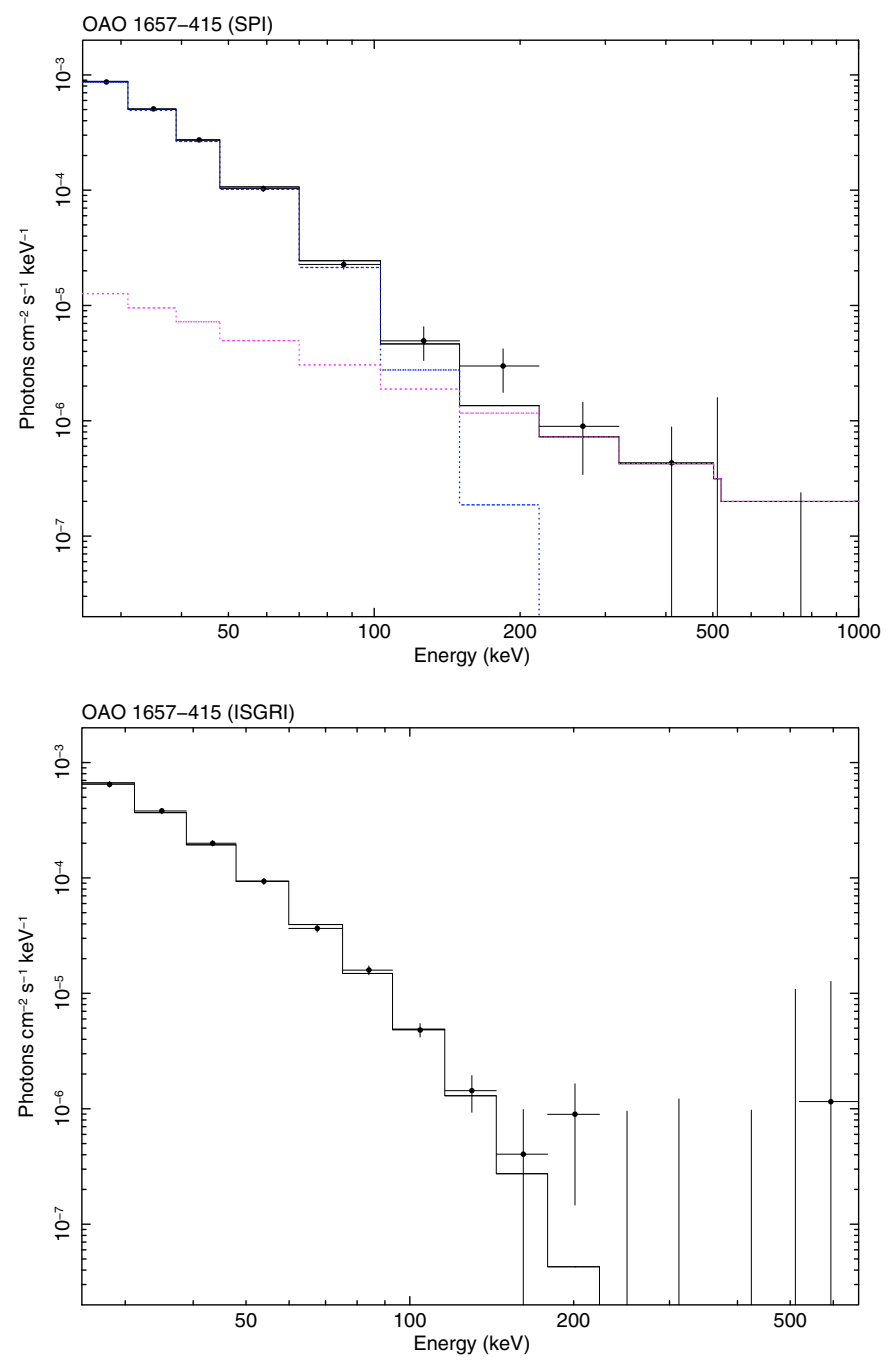

Fig. 10. The unfolded spectra of OAO 1657-415 derived from dataset 7 (see Table 1) with statistical errors: a) (top) SPI spectrum fit with powerlaw with exponential cutoff and additional powerlaw component b) (bottom) ISGRI spectrum fit with powerlaw with exponential cutoff (with increased errors, see text). The fit parameters are given in Sect. 4.9.

Norm $=0.23 \pm 0.21$ which is well compatible with the SPI data. This spectrum in shown in Fig. 10b.

\subsection{GX 339-4}

The microquasar GX 339-4 in one of the classical black hole X-ray binaries and has been studied by all X-ray and soft gamma-ray observatories, also with Swift (Tomsick et al. 2008) and INTEGRAL (see Joinet et al. 2007, who also give a review of the literature). Like other black hole binaries, the source has two main states, the low-hard state during which the high-energy spectrum is essentially a powerlaw with index $\Gamma=1.4-2.1$ with an exponential cutoff at hundred to a few hundred $\mathrm{keV}$, and the high-soft state during which $\Gamma$ goes to values $\geq 2.4$ but without cutoff (see again Joinet et al. 2007). Studies of the soft-gamma ray emission with OSSE have also found emission up to $400 \mathrm{keV}$ (Grabelsky et al. 1995). Joinet et al. (2007) found evidence for emission above $200 \mathrm{keV}$ in the low-hard state at the $5 \sigma$ level.

Our time-averaged spectrum should be a superposition of all states of the object. As a first step, we fit a simple powerlaw to the data and obtain index $\Gamma=2.08 \pm 0.04$, Norm $=0.35 \pm 0.05$ 

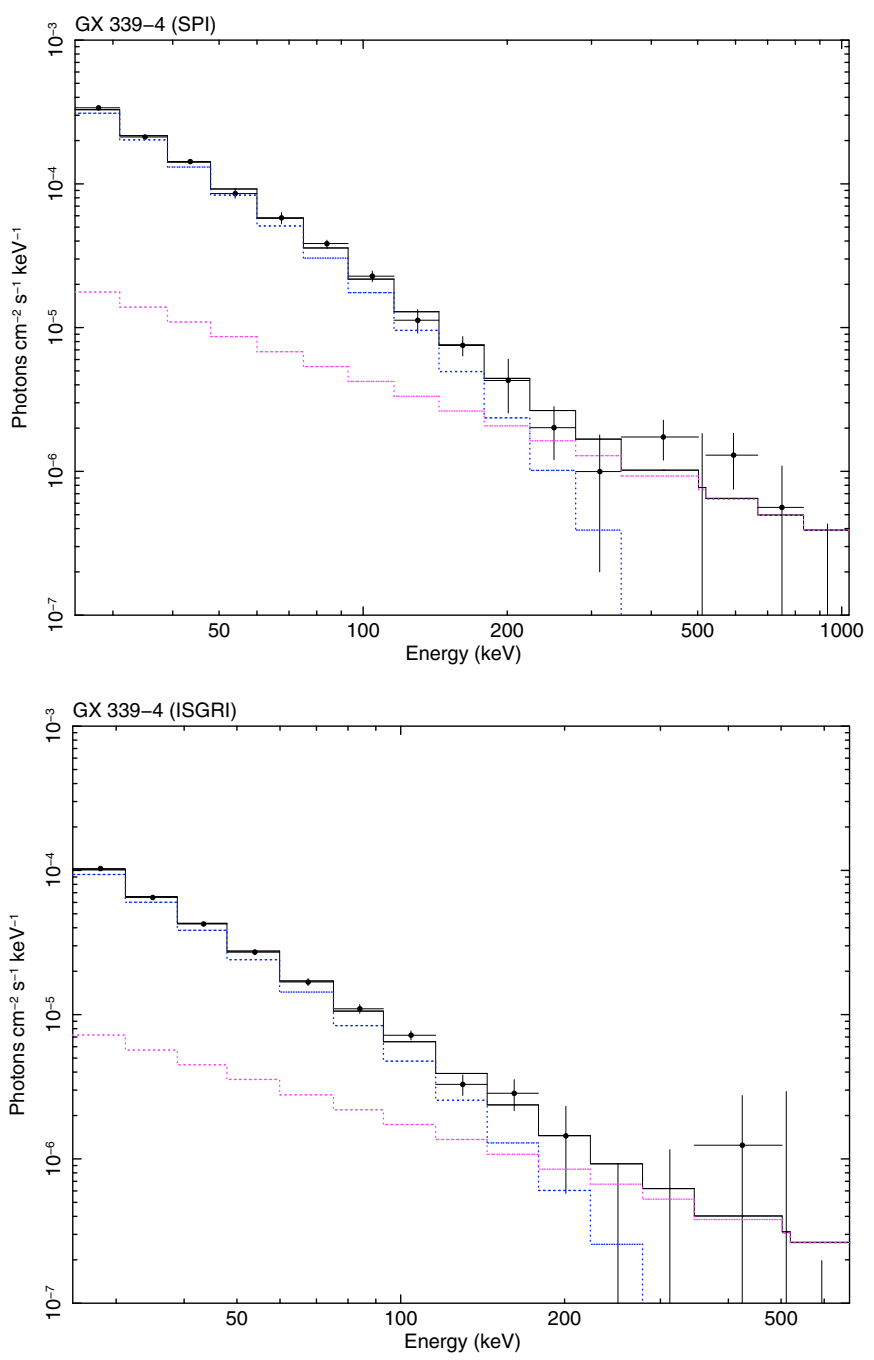

Fig. 11. The unfolded spectra of GX 339-4 derived from dataset 8 (see Table 1) with statistical errors: a) (middle) SPI spectrum fit with twocomponent model: powerlaw with exponential cutoff and second powerlaw. b) (Bottom) ISGRI spectrum fit with the same model as in a). The fit parameters are given in Sect. 4.10.

with a reduced $\chi^{2}$ of 1.2 for 15 d.o.f. The residuals are systematically negative from $100-300 \mathrm{keV}$ but seem to be systematically positive above $300 \mathrm{keV}$ indicating a hard tail. The significance of the emission above $200 \mathrm{keV}$ is $6.4 \sigma$.

The same fit applied to the ISGRI data gives a good reduced $\chi^{2}$ of 0.6 for 13 d.o.f. and best fit parameters $\Gamma=2.07 \pm$ 0.04 and Norm $=0.10 \pm 0.01$ confirming the shape of the SPI result up to $200 \mathrm{keV}$. Beyond $200 \mathrm{keV}$, the ISGRI result is not sensitive enough. As in the SPI spectrum, there is no evidence for a cutoff. A hard tail cannot be ruled out by the ISGRI data.

In order to improve our fit to the SPI data and to better be able to compare with previous measurements, we fit a more complex model consisting of two components: (a) a powerlaw with exponential cutoff (in our energy range this is equivalent to a thermal Comptonisation model, one of the main candidate emission mechanisms for microquasars, see Sect. 4.6) and (b) an additional powerlaw in order to describe a potential hard tail which may obscure the observation of a cutoff in our spectrum.

Since we lack the soft X-ray data to better constrain the cutoff energy, we fix it to $100 \mathrm{keV}$ consistent with previous measurements (if $E_{\text {cutoff }}$ is permitted to vary freely, we get a best fit value of $88 \pm 56 \mathrm{keV}$ ). Figure 11a shows the result of this fit. The best fit parameters are $\Gamma_{1}=1.6 \pm 0.1$, Norm $_{1}=$ $0.09 \pm 0.03, \Gamma_{2}=1.0 \pm 0.6, \operatorname{Norm}_{2}($ Flux at $200 \mathrm{keV})=$ $(3.8 \pm 2.4) \times 10^{-6} \mathrm{~cm}^{-2} \mathrm{~s}^{-1} \mathrm{keV}^{-1}$ with an improved reduced $\chi^{2}$ of 0.8 for 13 d.o.f.

The index $\Gamma_{1}$ of the dominant powerlaw at lower energies is well consistent with that observed by Tomsick et al. (2008) up to $100 \mathrm{keV}$. The additional powerlaw becomes dominant at about $200 \mathrm{keV}$. This confirms the feature already noted by Joinet et al. (2007) who speculated that it could be produced in a jet.

The more complex model is also fully compatible with the ISGRI data. Fitting the two-component model with fixed $E_{\text {cutoff }}=100 \mathrm{keV}$ and $\Gamma_{2}=1.0$ to the ISGRI data, we obtain a reduced $\chi^{2}$ of 0.7 for 12 d.o.f. and best fit parameters $\Gamma_{1}=$ $1.7 \pm 0.1$ (Fig. 11b).

\subsection{1. $4 U 1700-377$}

The eclipsing, 3.4 day X-ray binary 4U 1700-377 consists of a hot, bright supergiant and very probably a neutron star because its overall X-ray spectrum is similar to that of an accreting pulsar. The system has been studied in detail by many authors. For a review see e.g. van der Meer et al. (2005). In soft X-rays, the source displays a multitude of line features. The high energy spectrum has been modelled successfully by a powerlaw with high energy cutoff with additional photoelectric absorption for the points below $\approx 25 \mathrm{keV}$. Authors have also employed more complex models like thermal bremsstrahlung or thermal Comptonisation. In all cases, even though the source is strongly variable, the spectral shape above about $25 \mathrm{keV}$ was found to be independent of the flux state.

Our SPI spectrum is indeed well described by a powerlaw with high energy cutoff (Xspec model HIGHECUT). Figure 12a shows the SPI spectrum. The best fit parameters are $\Gamma=2.3 \pm$ 0.1 , Norm $=5.0 \pm 2.4, E_{\mathrm{c}}=(31 \pm 2) \mathrm{keV}, E_{\mathrm{f}}=(65 \pm 11) \mathrm{keV}$. The reduced $\chi^{2}$ is 0.9 for 7 d.o.f.

This agrees well with the literature: e.g. Maisack et al. (1994) find $\Gamma=2.55_{-0.55}^{+0.15}, E_{\mathrm{c}}=\left(20.1_{-20.1}^{+11.4}\right) \mathrm{keV}, E_{\mathrm{f}}=\left(69.8_{-38.3}^{+9.7}\right) \mathrm{keV}$. But also a simpler power law with exponential cutoff at $48 \pm 4 \mathrm{keV}$ and $\Gamma=2.0 \pm 0.1$ describes the data reasonably.

As for the other two sources in dataset 7, the statistical errors for the ISGRI spectrum of 4 U 1700-377 (Fig. 12b) had to be increased by $5 \%$ of the flux. This is certainly at least partially caused by the strong variability of 4 U 1700-377 (see also Sect. 3.1.3). A fit with a power law and exponential cutoff gives the best fit parameter values $\Gamma=2.3 \pm 0.2$, Norm $=7 \pm 3$, $E_{\text {cutoff }}=(74 \pm 14) \mathrm{keV}$, in agreement with SPI. The reduced $\chi^{2}$ is 0.9 for 12 d.o.f.

\subsection{IGR J17091-3624}

The object IGR J17091-3624 was discovered in 2003 by INTEGRAL (Kuulkers et al. 2003) and subsequently studied by a small number of authors based on archival data (Revnivtsev et al. 2003b) and INTEGRAL and RXTE data (Capitanio et al. 2006b). Chaty et al. (2008) recently found that the most probable candidate for a companion star is neither a giant nor a supergiant. They conclude that the system is most probably a LMXB. The source is variable but weak ( $\leq 10 \mathrm{mCrab}$ above $25 \mathrm{keV})$. The spectrum above a few ten $\mathrm{keV}$ is in good approximation a hard powerlaw with index varying between 1.6 and 2.2, but has also been successfully modelled with thermal Comptonisation (Capitanio et al. 2006b). 

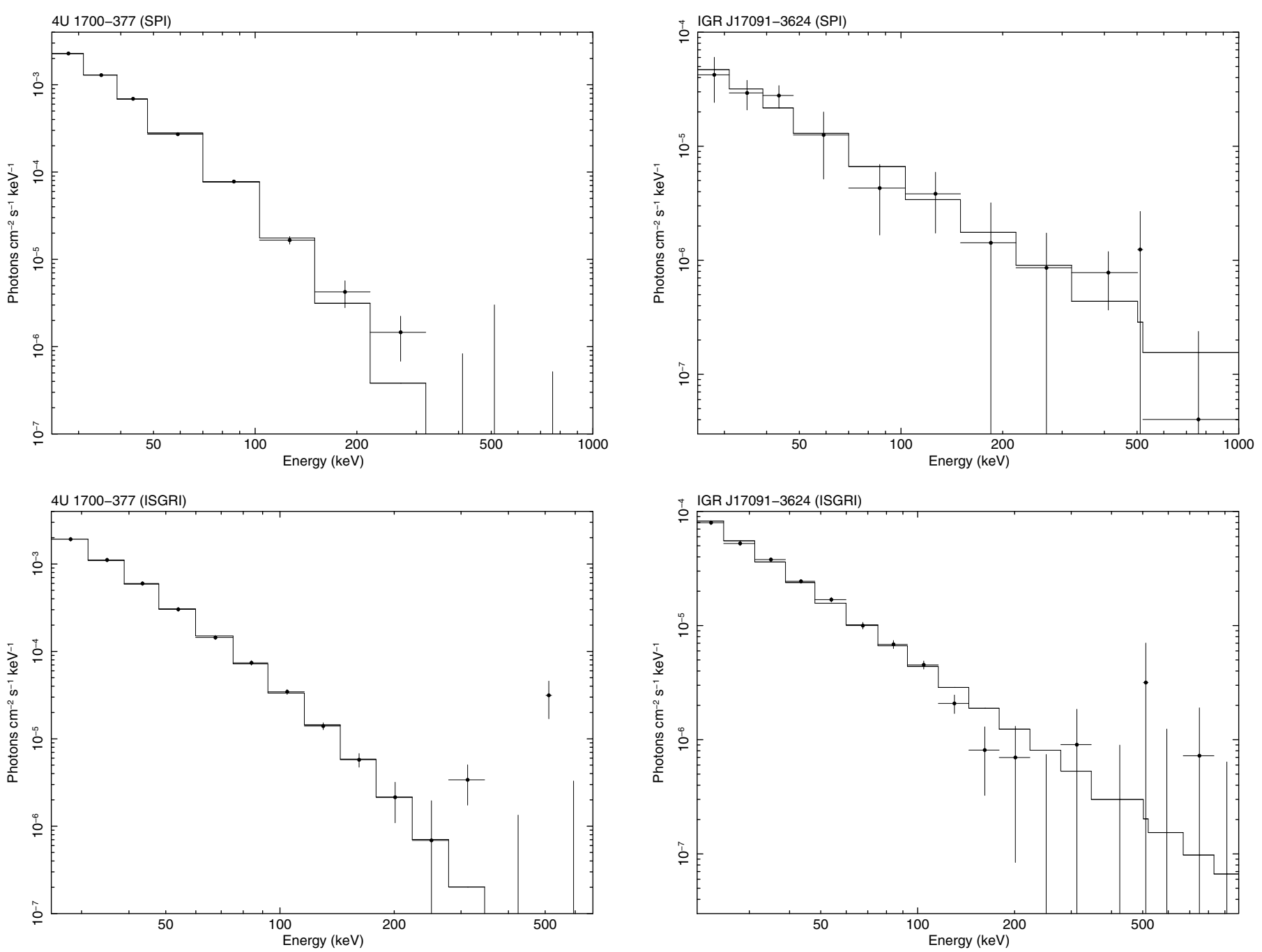

Fig. 12. The unfolded spectra of $4 \mathrm{U}$ 1700-377 derived from dataset 7 (see Table 1) with statistical errors: a) (top) SPI spectrum; b) (bottom) ISGRI spectrum. Spectral model is in both cases a powerlaw with high energy cutoff. The fit parameters are given in Sect. 4.11.

Fig. 13. The unfolded spectra of IGR J17091-3624 derived from dataset 9 (see Table 1) with statistical errors: a) (top) SPI spectrum with powerlaw fit; b) (middle) ISGRI spectrum with powerlaw fit. The fit parameters are given in Sect. 4.12.

\subsection{3. $G \times 354-0$}

When fitting our time-averaged SPI spectrum of IGR J17091-3624 (Fig. 13a), we find that a simple powerlaw describes it well. We find as best fit parameters $\Gamma=1.8 \pm$ 0.2 , Norm $=0.02 \pm 0.01$ with a reduced $\chi^{2}$ of 0.4 for 9 d.o.f. This is consistent with the previous measurements mentioned above and with the notion that the source was mostly in the low-hard state during our observation time.

Fitting a powerlaw to the corresponding ISGRI spectrum (Fig. 13b) gives a reduced $\chi^{2}$ of 1.2 for 16 d.o.f., $\Gamma=1.93 \pm 0.04$, and Norm $=0.034 \pm 0.005$ consistent with the SPI spectrum.

In order to compare our spectra with the ISGRI spectra by Captanio et al. (2006b), we also fit a thermal Comptonisation model (Xspec model COMPTT). We follow Captanio et al. and fix the soft photon temperature $k T_{0}=0.1 \mathrm{keV}$. From our ISGRI spectrum we then obtain a plasma optical depth $\tau_{\mathrm{p}}=$ $1.5 \pm 0.3$, and a plasma temperature $k T=(36 \pm 8) \mathrm{keV}$, Norm $=$ $0.0016 \pm 0.0005$, and a reduced $\chi^{2}$ of 0.5 for 12 d.o.f. (Fig. 13c). This is consistent with the values found by Captanio et al. (2006b).

The LMXB GX 354-0, also known as 4U 1728-34, consists of an accreting neutron star and probably a main sequence star and has been classified as a bursting atoll source which implies significant spectral variability. It has been studied by most X-ray observatories including INTEGRAL (see Falanga et al. 2006, and references therein). Above $25 \mathrm{keV}$, the spectrum has been typically modelled with a thermal Comptonisation spectrum. However, the studies do not extend much beyond $100 \mathrm{keV}$.

In our spectral analysis of the time-averaged spectrum, we initially fit a simple powerlaw. For the SPI data, this already gives a satisfactory fit (Fig. 14a) with a reduced $\chi^{2}$ of 0.7 for 9 d.o.f. and best fit parameters $\Gamma=3.26 \pm 0.06$ and Norm $=$ $26.4 \pm 5.9$. The same is true if we fit a powerlaw to the ISGRI data (Fig. 14b): we obtain a reduced $\chi^{2}$ of 1.0 for 13 d.o.f., and parameters $\Gamma=3.53 \pm 0.02$, Norm $=43.3 \pm 3.0$, steeper than the SPI spectrum.

Falanga et al. (2006) find that a thermal Comptonisation model (Xspec model COMPTT) describes the data well. Lacking soft X-ray information we take the values for the soft photon temperature $k T_{0}$ and the plasma temperature $k T$ from the recent measurement by Falanga et al. (for the low-hard state), 

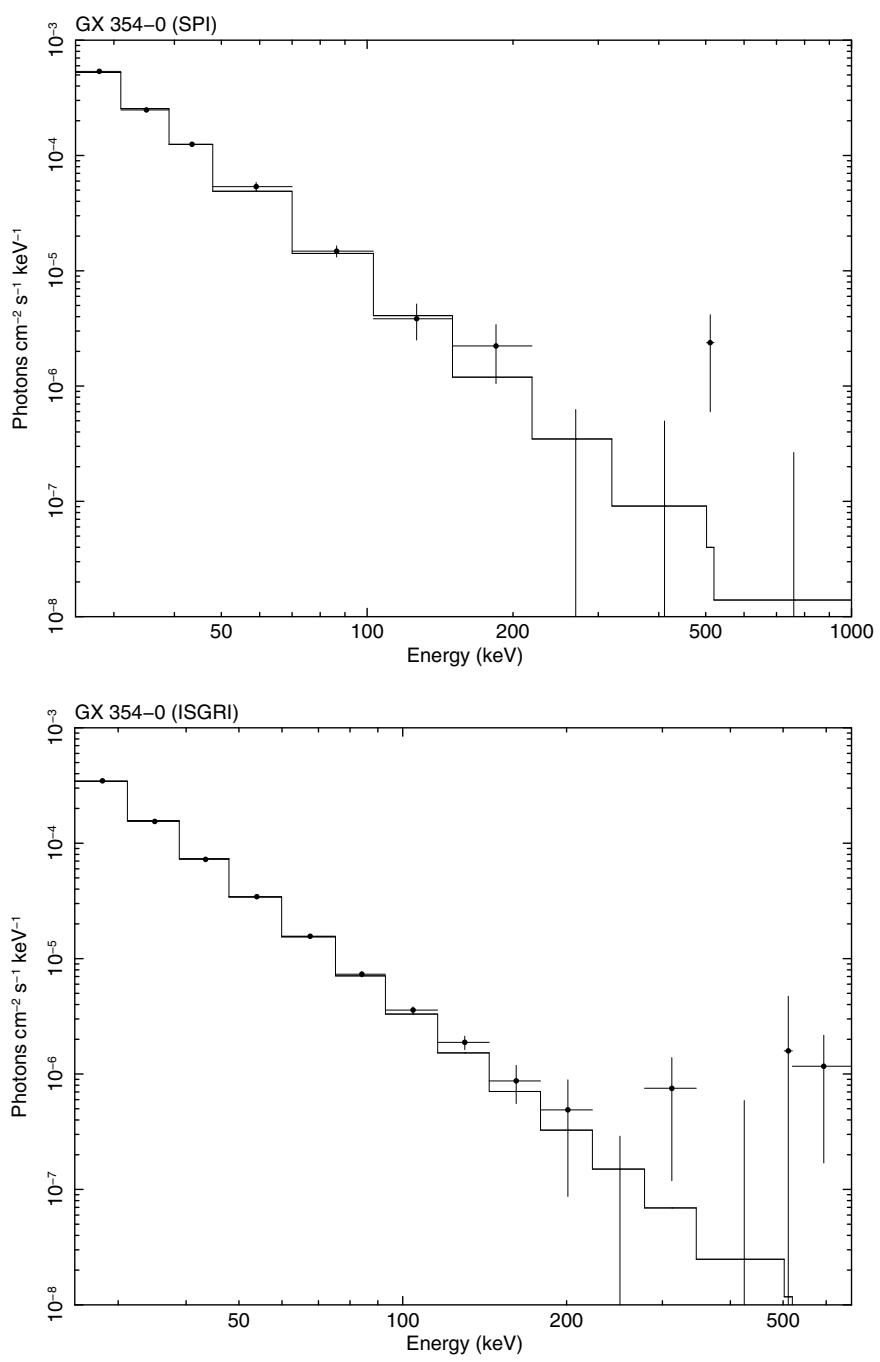

Fig. 14. The unfolded spectra of GX 354-0 derived from dataset 9 (see Table 1) with statistical: a) (top) SPI spectrum with powerlaw fit; b) (bottom) ISGRI spectrum fit with powerlaw. The fit parameters are given in Sect. 4.13. The combined significance of the emission above $200 \mathrm{keV}$ from the SPI and ISGRI data is $3 \sigma$.

$k T_{0}=1.18 \mathrm{keV}$ and $k T=35 \mathrm{keV}$, and fix them in our fit. Applied to the SPI data this results in a reduced $\chi^{2}$ of 1.9 for 9 d.o.f. and unevenly scattered residuals suggesting a hard tail. However, the sensitivity of our measurement does not permit a more detailed study of this feature. Also the ISGRI data are not compatible with the pure COMPTT spectrum proposed by Falanga et al. Here we get a reduced $\chi^{2}$ of 7.6 for 11 d.o.f. even if we let all model parameters vary freely.

We conclude that our data are not well compatible with a pure thermal Comptonisation spectrum since it underpredicts the high-energy emission. The combined significance of the emission above $200 \mathrm{keV}$ from GX 354-0 is, however, only $3 \sigma$. Possibly, the averaging over several spectral states mimics a power law.

\subsection{4. $1 E$ 1740.7-2942}

The black hole candidate 1E 1740.7-2942 is a bright persistent source very near the Galactic Centre and the first source to be classified as a microquasar (see Bosch-Ramon et al. 2006, for a recent review). Its classification as a LMXB is controversial. Like other BHCs, 1E 1740.7-2942 exhibits low-hard and
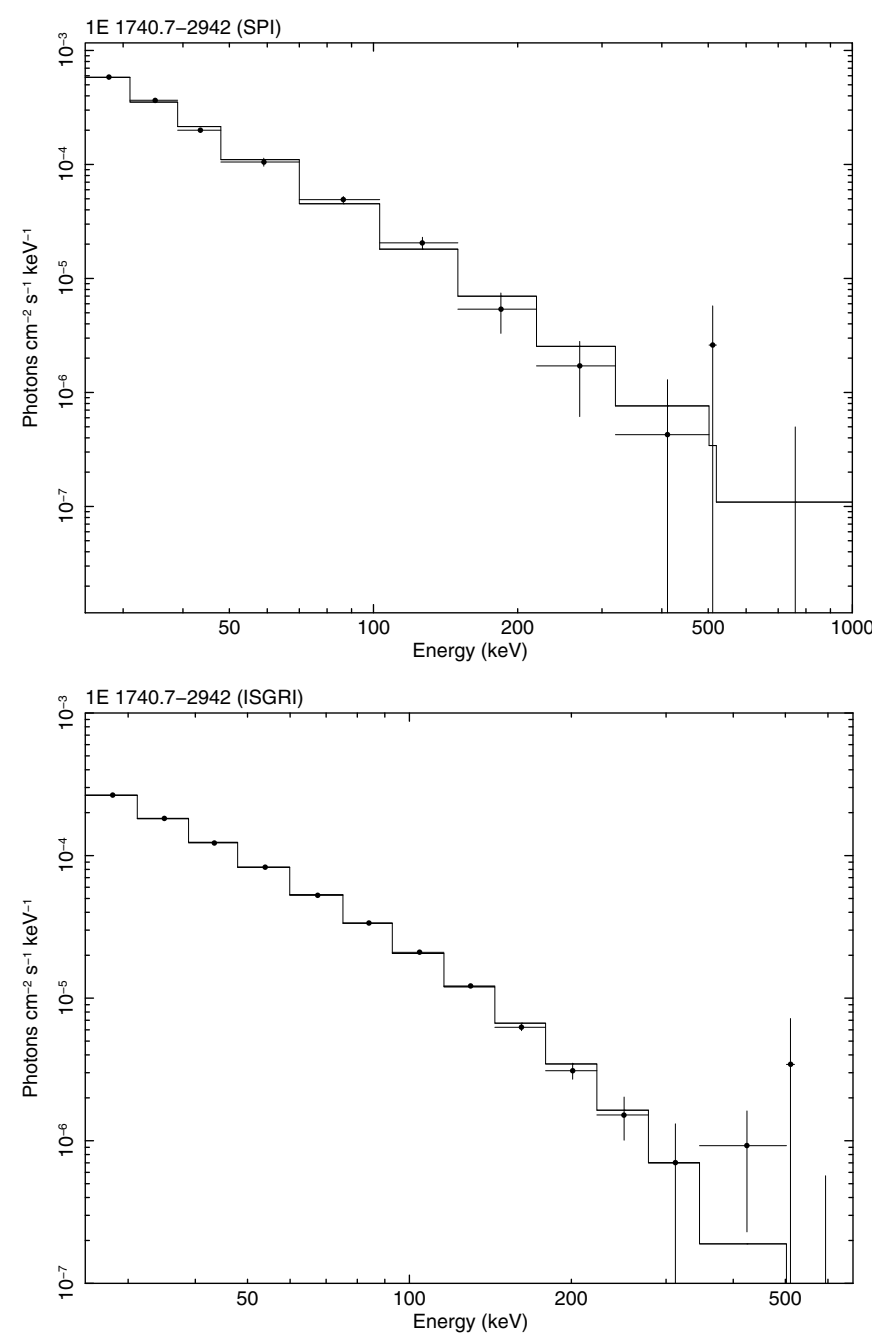

Fig. 15. The unfolded spectra of 1E 1740.7-2942 derived from dataset 9 (see Table 1) with statistical errors: a) (top) SPI spectrum; b) (bottom) ISGRI spectrum, in both cases fit by a powerlaw with exponential cutoff. The fit parameters are given in Sect. 4.14.

high-soft states. Using ISGRI data, Del Santo et al. (2005) find that the average spectrum above $25 \mathrm{keV}$ is approximately described by a powerlaw of index $\Gamma=2.3$. In individual periods they find harder spectra $(\Gamma \approx 1.5)$ with exponential cutoffs around $100 \mathrm{keV}$ which can alternatively be described with thermal Comptonisation models.

Bouchet et al. (1991), using GRANAT/SIGMA data, have reported a transient additional component (modelled by a Gaussian with $F W H M$ of $240 \mathrm{keV}$ ) peaking at $480 \mathrm{keV}$ which appeared for about a day in the spectrum and then disappeared. This observation has not yet been confirmed and our study would not pick up on such features since we are averaging over much longer timescales (unless such events would occur frequently).

We fit our SPI spectrum with a powerlaw and exponential cutoff (Fig. 15a) and obtain a reduced $\chi^{2}$ of 1.4 for 10 d.o.f. with $\Gamma=2.17 \pm 0.14$ in agreement with the average spectrum by Del Santo et al. (2005). The normalisation is Norm $=0.9 \pm 0.4$. The cutoff energy is a badly constrained $434 \pm 400 \mathrm{keV}$.

The same model fit to the ISGRI data gives best fit parameters of $\Gamma=1.44 \pm 0.04, E_{\text {cutoff }}=(113 \pm 8) \mathrm{keV}$, Norm $=0.040 \pm$ 0.005 , and a reduced $\chi^{2}$ of 0.7 for 12 d.o.f.

The ISGRI spectrum is in agreement with spectra found by Del Santo et al. (2005) (see above) but the agreement with SPI is at best marginal. In order to reconcile the two results, we fix 

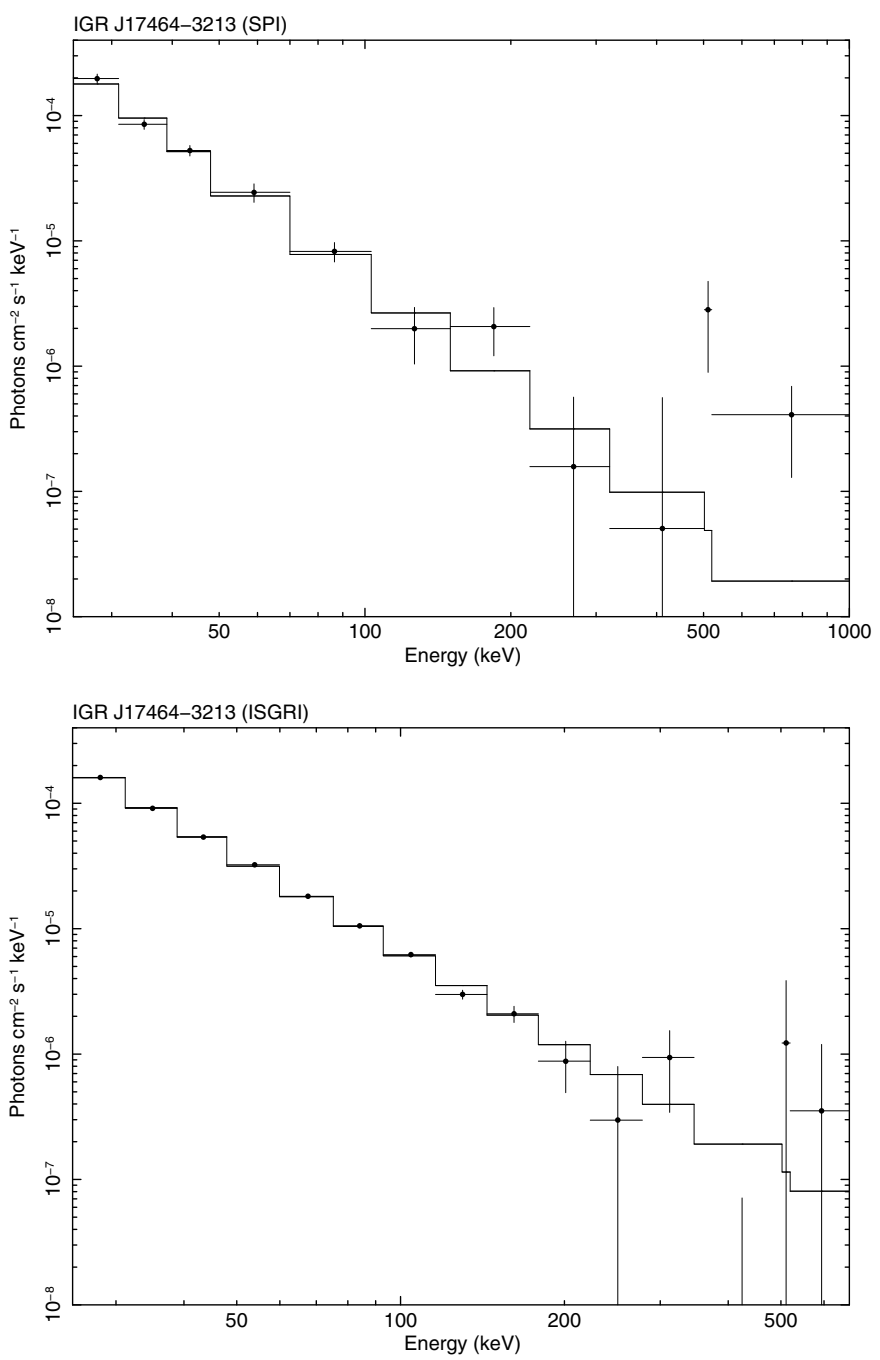

Fig. 16. The unfolded spectra of IGR J17464-3213 (a.k.a. H 1743-322) derived from dataset 9 (see Table 1) with statistical errors: a) (top) SPI spectrum with powerlaw fit; b) (bottom) ISGRI spectrum with same model as a). The fit parameters are given in Sect. 4.15. The significance of the emission above $200 \mathrm{keV}$ is $3 \sigma$.

the exponential cutoff in the model for the SPI data at the value determined by ISGRI. We obtain a reduced $\chi^{2}$ of 1.9 for 9 d.o.f. and best fit parameters $\Gamma=1.80 \pm 0.06$, Norm $=0.28 \pm 0.06$. The residuals suggest an additional hard component. But our sensitivity is not good enough to warrant a more detailed study. The agreement of the SPI results with the fluxes reported by B08 is very good (see Table 5).

\subsection{IGR J17464-3213}

The transient IGR J17464-3213 was discovered by INTEGRAL in 2003 (Revnivtsev et al. 2003a) and soon identified as the counterpart of the HEAO $1 \mathrm{X}$-ray source $\mathrm{H}$ 1743-322. The source was subsequently studied by several authors at X-ray, optical and radio wavelengths, in particular with INTEGRAL (see Joinet et al. 2005, for a recent review). Evidence for jets was found and the source is now regarded to be a microquasar (Corbel et al. 2005). Timing and spectral properties of the object suggest that it is a LMXB containing a black hole of about $10 M_{\odot}$.

The RXTE/ASM lightcurve of IGR J17464-3213 (e.g. Capitanio et al. 2006a) shows that the source can be in strong outburst for months at a time. In between outbursts it returns to a very low level of emission and remains there for months. Using data from RXTE and INTEGRAL up to $150 \mathrm{keV}$, Joinet et al. (2005) have studied the complex spectral variability of the object (typical of BHCs): the source exhibits softening during flux increases followed by phases of constant spectrum in spite of intensity changes by factors $2-3$. Above a few ten $\mathrm{keV}$, the spectrum is described by a powerlaw with index $\Gamma$ varying between 1.0 and 2.6, and a not very well constrained exponential cutoff around $100 \mathrm{keV}$.

The ISGRI lightcurve (Courvoisier et al. 2008) for IGR J17464-3213 shows three strong outburst periods and otherwise a low level of emission such that our time-averaged spectrum should be a superposition of the low-hard and the highsoft state. When examining our SPI spectrum, we find evidence for increased uncertainties in the lowest two energy bins possibly due to to insufficient source region modelling at the lowest energies. To be on the safe side, we increase the statistical errors by $5 \%$ of the flux for the entire spectrum. Fitting the spectrum with a powerlaw (Fig. 16a), then leads to a reduced $\chi^{2}$ of 1.1 for 9 d.o.f. and best fit parameters $\Gamma=2.82 \pm 0.14$, Norm $=2.1 \pm 1.1$.

Fitting a powerlaw to the ISGRI spectrum of (Fig. 16c) gives a perfect reduced $\chi^{2}$ of 0.9 and best fit parameters $\Gamma=$ $2.49 \pm 0.02$ and Norm $=0.63 \pm 0.04$. There is no evidence for a cutoff. The powerlaw index is still in agreement with our SPI measurement.

\subsection{GRS $1758-258$}

The LMXB GRS $1758+258$ is one of the well known microquasars and regarded to be similar to $1 \mathrm{E} 1740.7-2942$. It is also a black hole candidate. For a recent review see e.g. Pottschmidt et al. (2006). The source is bright and can show outburst which are however mostly too short for SPI's sensitivity. B08 and our analysis assume it to be a constant emitter. In the low-hard state, which the source mostly assumes, the high energy spectrum can roughly be described by a hard powerlaw with an exponential cutoff around $100 \mathrm{keV}$. In terms of physical models, thermal Comptonisation models and models involving black body emission from a disk have been applied. The spectrum above $200 \mathrm{keV}$ was hardly studied.

We fit our SPI spectrum initially with a powerlaw with exponential cutoff and obtain a reduced $\chi^{2}$ of 1.7 for 14 d.o.f. (Fig. 17a) and well constrained best fit parameters $\Gamma=1.54 \pm$ $0.07, E_{\text {cutoff }}=(178 \pm 28) \mathrm{keV}$, Norm $=0.12 \pm 0.03$, in agreement with previous measurements.

The same spectral fit applied to the ISGRI data results in best fit parameters $\Gamma=1.36 \pm 0.02, E_{\text {cutoff }}=(123 \pm 5) \mathrm{keV}$, Norm $=$ $0.059 \pm 0.004$, and reduced $\chi^{2}=1.9$ for 12 d.o.f., in marginal agreement with the SPI result.

In both cases, the fit quality is not very good which may be the result of assuming the source to be constant (but we chose to follow B08 consistently). In the SPI spectrum, there is a hint of a hard tail in the residuals. In fact, we achieve a fit improvement (reduced $\chi^{2}=1.6$ for 13 d.o.f.) if we freeze the cutoff energy and add a powerlaw as a second component. However, the chance probability of the improvement is 0.2 .

\subsection{Ginga 1826-24}

The LMXB Ginga 1826-24 was discovered in 1988 and initially found similar in spectrum and variability to the BHC Cyg X-1. Not much later, the relatively low cutoff energy $(\approx 58 \mathrm{keV})$ of 

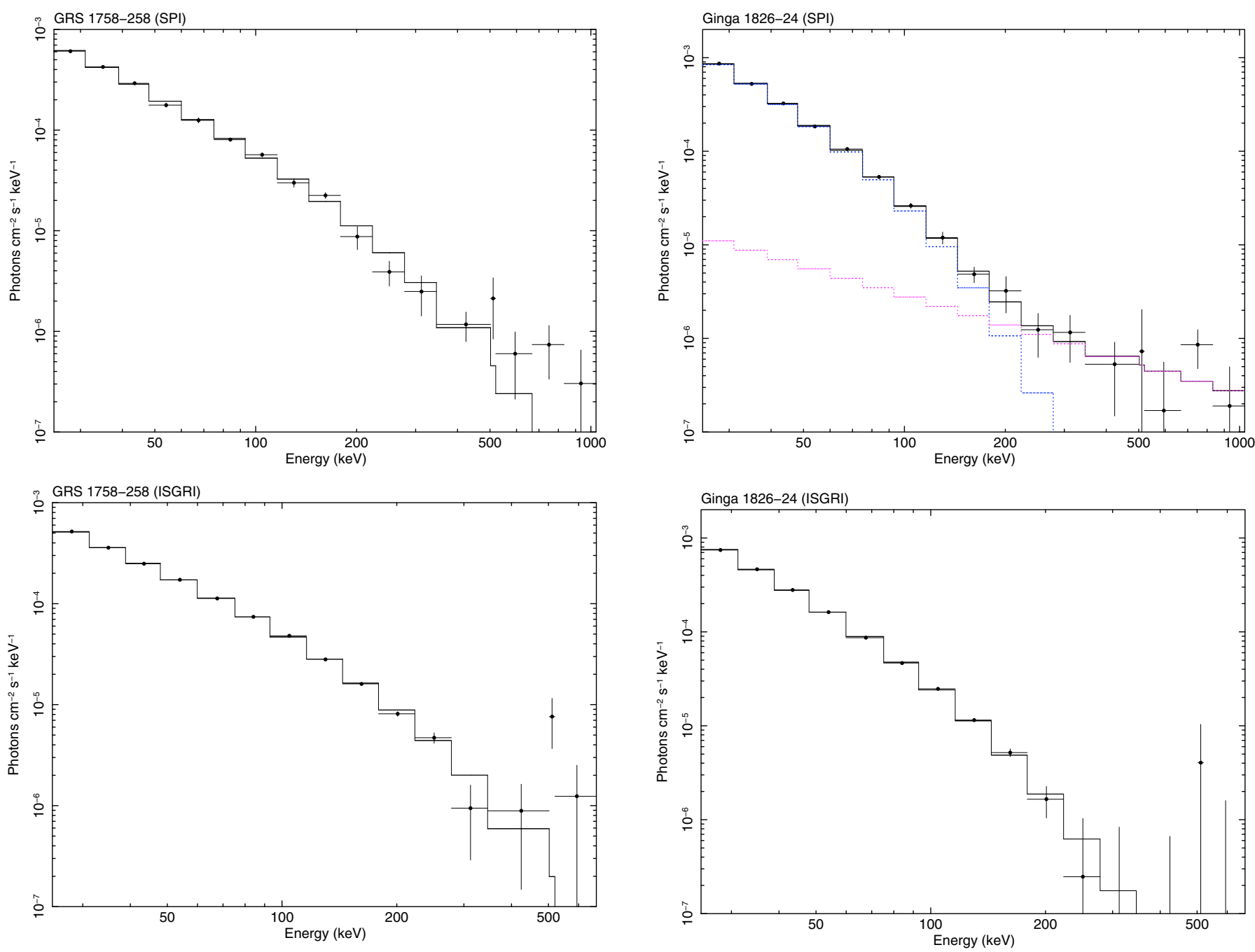

Fig. 17. The unfolded spectra of GRS 1758-258 derived from dataset 10 (see Table 1) with statistical errors: a) (top) SPI spectrum; and b) (bottom) ISGRI spectrum, both fit by powerlaw with exponential cutoff. The fit parameters are given in Sect. 4.16.

its powerlaw spectrum above a few ten $\mathrm{keV}$ and the observation of X-ray bursts led to the classification as a NS. The exceptional precision of the reoccurrence of the bursts (period $\approx 3.5 \mathrm{~h}$ ) also led to the nickname "clocked burster". For a recent review of the source properties see Thompson et al. (2005). The timeaveraged high-energy spectrum up to about $300 \mathrm{keV}$ was measured by Strickman et al. (1996a) and modelled as a powerlaw with exponential cutoff. They found $\Gamma=1.59 \pm 0.02$ and $E_{\text {cutoff }}=(57 \pm 8) \mathrm{keV}$.

Fitting our SPI spectrum of Ginga 1826-24 with a powerlaw with exponential cutoff, we obtain a reduced $\chi^{2}$ of 1.2 for 14 d.o.f. and best fit parameters $\Gamma=1.7 \pm 0.1, E_{\text {cutoff }}=(60 \pm$ 7) $\mathrm{keV}$, Norm $=0.36 \pm 0.10$ in good agreement with Strickman et al. (1996a).

The residuals show evidence for a hard tail above $200 \mathrm{keV}$. Accommodating the possibility of such a tail by adding a powerlaw component to the model, we obtain from the fit to the SPI data (Fig. 18a) a reduced $\chi^{2}$ of 0.5 for 12 d.o.f. and best fit parameters Norm $_{1}=0.24 \pm 0.09, \Gamma_{2}=0.8 \pm 0.69$, Norm $_{2}$ $($ at $200 \mathrm{keV})=(2.1 \pm 1.8) \times 10^{-6} \mathrm{~cm}^{-2} \mathrm{~s}^{-1} \mathrm{keV}^{-1}$. The second powerlaw component becomes dominant above ca. $300 \mathrm{keV}$.

Applying the same modelling to the ISGRI data (Fig. 18b), we obtain for a simple powerlaw with exponential cutoff a

Fig. 18. The unfolded spectra of Ginga 1826-24 derived from dataset 10 (see Table 1) with statistical errors: a) (top) SPI spectrum fit with twocomponent model consisting of a powerlaw with exponential cutoff and a second powerlaw; b) (bottom) ISGRI spectrum fit with powerlaw with exponential cutoff. The fit parameters are given in Sect. 4.17.

reduced $\chi^{2}$ of 1.5 for 12 d.o.f. and best fit parameters $\Gamma=1.78 \pm$ $0.04, E_{\text {cutoff }}=(69 \pm 3) \mathrm{keV}$, Norm $=0.42 \pm 0.05$. The residuals don't show any posibility for improvement.

We conclude that our time averaged spectra agree well with previously published results. SPI and ISGRI data are compatible with each other. The evidence for a hard tail above $200 \mathrm{keV}$ is weak even though B08 still find $25 \mathrm{mCrab}$ at $200-600 \mathrm{keV}$. We find less than half of that flux.

\subsection{GRS $1915+105$}

The LMXB GRS $1915+105$ is one of the most studied galactic black holes. For a review see e.g. Fender \& Belloni (2004) or Rodriguez et al. (2008a). The object is a microquasar, the first to exhibit superluminal motion in its jets, with a relatively large black hole mass of $\approx 14 M_{\odot}$, a large binary period of $33.5 \mathrm{~d}$, and an extremely complex time variability which is attributed to accretion disk instabilities (Greiner et al. 1996; Done et al. 2004).

GRS $1915+105$ was extensively monitored by INTEGRAL. A detailed study of two years of the INTEGRAL observations 

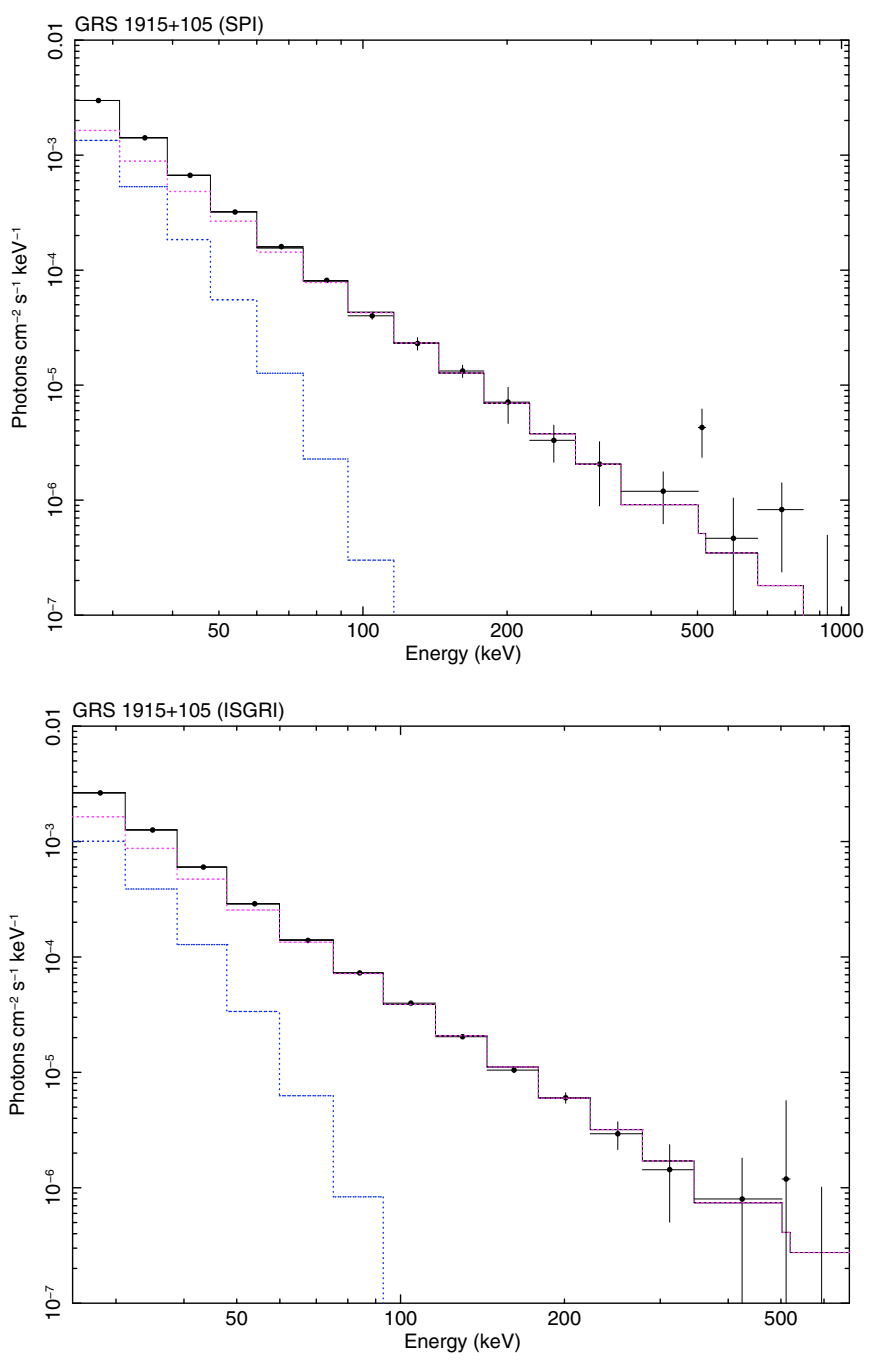

Fig. 19. The unfolded spectra of GRS $1915+105$ derived from dataset 11 (see Table 1) with statistical errors: a) (top) SPI spectrum fit with two-component model consisting of powerlaw with exponential cutoff and second powerlaw; b) (bottom) ISGRI spectrum fit by same model as used in a). The fit parameters are given in Sect. 4.18.

was published in Rodriguez et al. (2008a, 2008b). They analyse ISGRI spectra up to $300 \mathrm{keV}$ and distinguish several spectral states which they fit with a number of multi-component models involving for the high energy part thermal Comptonisation (Xspec model COMPTT) with seed photon temperatures between 0.7 and $2.1 \mathrm{keV}$ and a powerlaw with index between 2.0 and 2.9 .

Our time-averaged spectrum of the source is a superposition of all spectral states. As for the previous sources we initially try to fit a powerlaw with exponential cutoff. This results in a high reduced $\chi^{2}$ of 2.4 for 14 d.o.f., a photon index of $3.30 \pm 0.02$, and a cutoff energy far beyond the upper end of our energy range. The residuals indicate that a more sophisticated model is needed.

Next we try a powerlaw with exponential cutoff with an additional powerlaw. This results in a reduced $\chi^{2}$ of 0.6 for 12 d.o.f. and badly constrained best fit parameters $\Gamma_{1}=1.7 \pm 1.7, E_{\text {cutoff }}=$ $(13 \pm 9) \mathrm{keV}, \Gamma_{2}=2.8 \pm 0.2$, Norm $_{1}=3.7 \pm 16$, and Norm $_{2}$ $($ at $200 \mathrm{keV})=(1.4 \pm 0.1) \times 10^{-5} \mathrm{~cm}^{-2} \mathrm{~s}^{-1} \mathrm{keV}^{-1}$, i.e. a dominant pure powerlaw with a weak additional low-energy component becoming negligible at a few ten $\mathrm{keV}$.
Applying the same model to the ISGRI data results in $\Gamma_{2}=2.87 \pm 0.05$ and $\operatorname{Norm}_{2}($ at $200 \mathrm{keV})=1.17 \pm 0.04 \times$ $10^{-5} \mathrm{~cm}^{-2} \mathrm{~s}^{-s} \mathrm{keV}^{-1}$ for the dominant component and $\Gamma_{1}=0.8 \pm$ $1.0, E_{\text {cutoff }}=8 \pm 2 \mathrm{keV}$, Norm $_{1}=0.09 \pm 0.25$ for the weak lowenergy component. The reduced $\chi^{2}$ is 0.7 for 10 d.o.f. The agreement with the SPI result is good.

\subsection{Cyg $X-1$}

The HMXB Cyg X-1 consists of a black hole candidate of high but uncertain mass and an OB supergiant (see Gies et al. 2003; Ziolkowski 2005, and references therein). It is one of the brightest hard X-ray sources and has been studied extensively by many authors in all wavebands. For a brief review see e.g. Szostek \& Zdziarski (2007) and Shaposhnikov \& Titartchuk (2006) and references therein. The radio emission shows evidence of a moderately relativistic jet. The object has therefore recently been called a microquasar (e.g. Russell et al. 2007).

Long-term monitoring has revealed two main spectral states like in other $\mathrm{BH}$ binaries, the low-hard state and the high-soft state, between which the source transits. It spends the dominant fraction of time in the low-hard state (Zhang et al. 1997; Hjalmarsdotter et al. 2008). Our time-averaged spectrum of Cyg X-1 should therefore be more similar to the low hard state which is roughly a hard powerlaw ( $\Gamma$ between 1.4 and 2.1 ) with a break at $E>50 \mathrm{keV}$ (Cadolle Bel et al. 2006).

The X-ray spectra of individual states have been modelled up to $300 \mathrm{keV}$ by several authors with a variety of combinations of disk black body and thermal Comptonisation with additional Fe fluorescence line and reflection components (see again Cadolle Bel et al. 2006).

Lacking the soft X-ray information and averaging over all source states, we fit our SPI spectrum of Cyg X-1 initially with a simple powerlaw with exponential cutoff and obtain a very large reduced $\chi^{2}$ of 5.3 for 14 d.o.f. The best fit parameters $(\Gamma=1.65 \pm$ $0.01, E_{\text {cutoff }}=(180 \pm 5) \mathrm{keV}$, Norm $\left.=2.3 \pm 0.1\right)$ agree well with the expectations from previous measurements and, up to about $300 \mathrm{keV}$, the model describes the data reasonably well but there is a clear and very significant hard tail above $300 \mathrm{keV}$.

This hard tail in the low-hard state spectrum of Cyg X-1 was already observed up to about $5 \mathrm{MeV}$ with COMPTEL by McConnell et al. (2000). They did not observe an indication of a cutoff. It was also already studied with INTEGRAL by Cadolle Bel et al.(2006) and Malzac et al. (2006).

Adding a powerlaw to our model to accommodate the hard tail, but leaving all parameters free, we obtain a much better fit (Fig. 20a) with reduced $\chi^{2} 0.9$ for 12 d.o.f. and residuals which don't show any sign of systematic deviations. The best fit parameters are $\Gamma_{1}=1.3 \pm 0.2, E_{\text {cutoff }}=(95 \pm 15) \mathrm{keV}$, Norm $_{1}=$ $0.43 \pm 0.35, \Gamma_{2}=2.11 \pm 0.1, \operatorname{Norm}_{2}($ at $200 \mathrm{keV})=(9.9 \pm 1.8) \times$ $10^{-5} \mathrm{~cm}^{-2} \mathrm{~s}^{-1} \mathrm{keV}^{-1}$.

Applying the same model to the ISGRI data (Fig. 20b), we obtain a reduced $\chi^{2}$ of 1.5 for 10 d.o.f. and best fit parameters $\Gamma_{1}=1.44 \pm 0.14, E_{\text {cutoff }}=(115 \pm 18) \mathrm{keV}$, Norm $_{1}=0.9 \pm$ $0.5, \Gamma_{2}=2.2 \pm 0.14, \operatorname{Norm}_{2}($ at $200 \mathrm{keV})=(3.6 \pm 2.3) \times$ $10^{-5} \mathrm{~cm}^{-2} \mathrm{~s}^{-1} \mathrm{keV}^{-1}$, i.e. the best fit parameters are in agreement with those from SPI but are not as well constrained because the ISGRI spectrum is lacking high energy sensitivity.

To further compare with the COMPTEL data, we fit a powerlaw to our SPI data above $500 \mathrm{keV}$. We find an index of $1.9 \pm$ 0.5 which is much harder than the index $3.3 \pm 0.4$ measured by McConnell et al. (2000) in the neighbouring energy range. One explanation of this difference could be source variability, which 

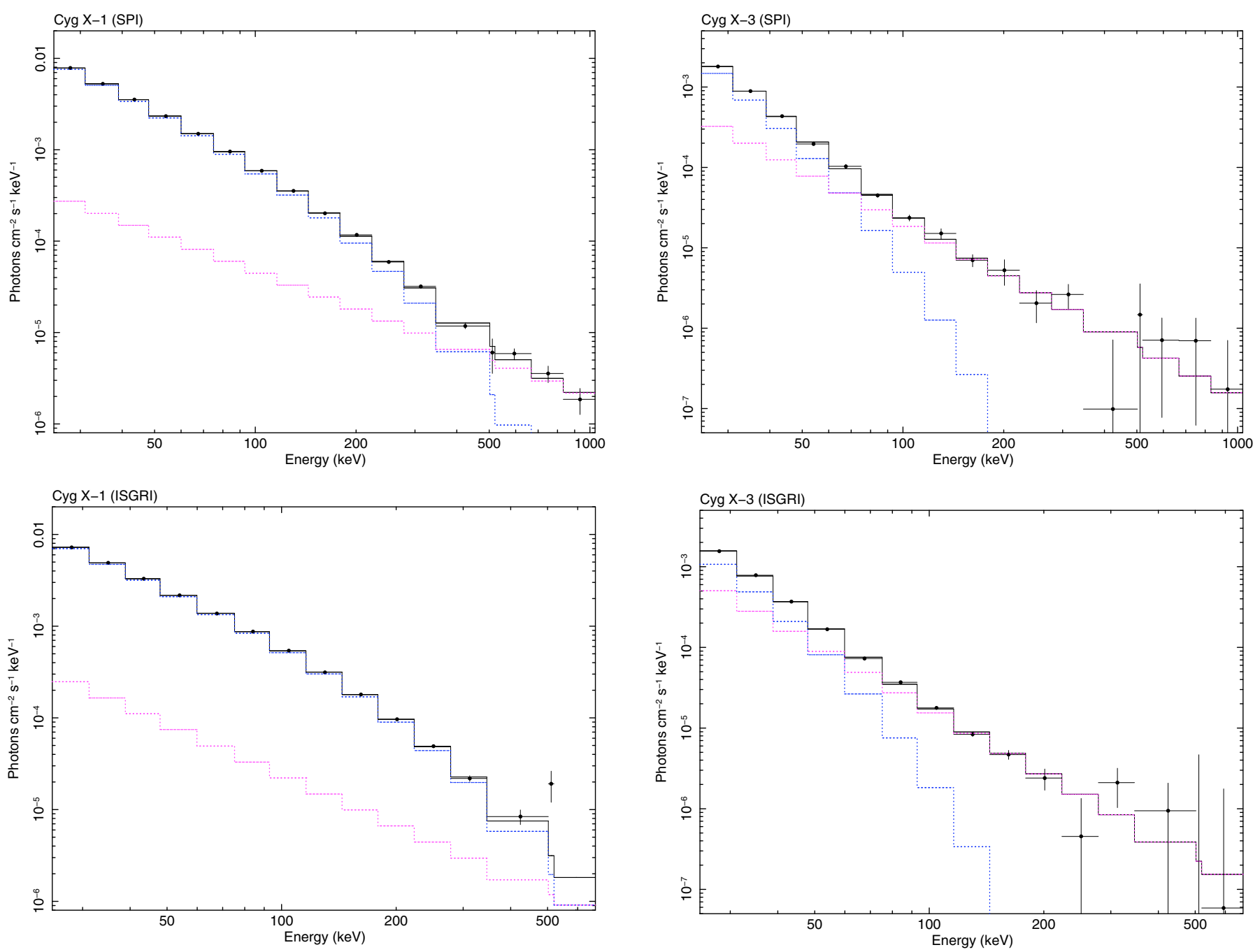

Fig. 20. The unfolded spectra of Cyg X-1 derived from dataset 12 (see Table 1) with statistical errors: a) (top) SPI spectrum fit with twocomponent model consisting of a powerlaw with exponential cutoff and a second powerlaw; b) (bottom) ISGRI spectrum fit by same model as in a). The fit parameters are given in Sect. 4.19.

has indeed been suggested to be present as a variable broad line feature around $1 \mathrm{MeV}$ (McConnell et al. 1989, 2000).

We conclude that even though our spectra have extremely high statistical significance, it is possible to model them with a phenomenological model with only five parameters consisting of a cutoff powerlaw and second powerlaw. The cutoff powerlaw can be interpreted as a contribution from thermal Comptonisation as already suggested by many previous authors (see above) while the hard tail is most likely a product of the jet.

\subsection{Cyg $X-3$}

Cyg X-3 is a HMXB with an unusually short binary period of $4.8 \mathrm{~h}$ and a Wolf-Rayet star as an unusual stellar companion. Despite its brightness and the intensive study at all wavebands over four decades, recently published articles still declare many of its properties as uncertain, in particular the nature of the compact object contained in it. Szostek \& Zdziarski (2008) and Hjalmarsdotter et al. (2008), H08 in the following, give a brief review of the literature. They also argue that the compact

Fig. 21. The unfolded spectra of Cyg X-3 derived from dataset 13 (see Table 1) with statistical errors: a) (top) SPI spectrum fit with twocomponent model consisting of a powerlaw with exponential cutoff and a second powerlaw; b) (bottom) ISGRI spectrum fit by same model as in a). The fit parameters are given in Sect. 4.20.

object in Cyg X-3 could be a quite massive black hole. The object has also been called a microquasar because of its radio jets (Marti et al. 2007). At lower energies, the intense stellar wind from the companion star leads to strong absorption features. Above $25 \mathrm{keV}$, however, the effect on the spectral shape is small (H08).

Cyg X-3 has a binodal distribution of soft X-ray count rates. The distribution of its spectral hardness, however, only shows one peak at low hardness with a long asymmetric tail to higher hardness (H08). The authors note that the soft X-ray hardness in the case of Cyg X-3 may be a bad indicator of the state of the source because of its strong absorption. The source probably still has a separate hard state at higher energies which it occupies roughly two thirds of the time.

The time-averaged spectra we are studying here should therefore be a superposition of spectra from all states, but more similar to the harder spectra.

We initially fit our SPI spectrum of Cyg X-3 with a powerlaw with exponential cutoff. This results in a relatively high reduced $\chi^{2}$ of 1.8 , an index $\Gamma=3.26 \pm 0.04$ and a cutoff 
beyond the upper end of our energy range. The residuals suggest a hard tail beginning at about $100 \mathrm{keV}$. Adding a powerlaw as second component, we obtain a reduced $\chi^{2}$ of 0.8 for 12 d.o.f. (Fig. 21a) but the fit parameters are poorly constrained $\left(\Gamma_{1}=\right.$ $2.0 \pm 0.7, E_{\text {cutoff }}=(20 \pm 10) \mathrm{keV}$, Norm $_{1}=3.5 \pm 6.6, \Gamma_{2}=2.4 \pm$ $0.3, \operatorname{Norm}_{2}($ at $\left.200 \mathrm{keV})=(9.0 \pm 1.1) \times 10^{-6} \mathrm{~cm}^{-2} \mathrm{~s}^{-1} \mathrm{keV}^{-1}\right)$ because the best fit cutoff energy is outside our energy range. The powerlaw component without cutoff constitutes the dominant part of the emission above a few $10 \mathrm{keV}$. There is no evidence for a cutoff at higher energies, but our data points become insignificant above $350 \mathrm{keV}$.

An unusually low cutoff energy implying a most likely plasma temperature of only $4 \mathrm{keV}$ was also found in the INTEGRAL data analysis by $\mathrm{H} 08$ when assuming a thermal Comptonisation model.

When inspecting our ISGRI spectrum of Cyg X-3, we find evidence for enlarged systematic errors which are probably caused by the crowded field around the object including the nearby and extremely bright Cyg X-1. We increase the statistical errors by $0.5 \%$ of the flux. With this change, the fit of the same two-component model as for the SPI data with $E_{\text {cutoff }}$ fixed to $20 \mathrm{keV}$ results in a reduced $\chi^{2}$ of 1.7 for 11 d.o.f. and best fit parameters $\Gamma_{1}=2.00 \pm 0.08$, Norm $_{1}=3.4 \pm 0.7, \Gamma_{2}=2.7 \pm$ 0.2, Norm $_{2}($ at $200 \mathrm{keV})=(5.3 \pm 0.5) \times 10^{-6} \mathrm{~cm}^{-2} \mathrm{~s}^{-1} \mathrm{keV}^{-1}$ (Fig. 21b) in agreement with the SPI results.

\subsection{Systematic errors of flux measurements in crowded fields}

As described in Sect. 3.3 we derive our systematic error estimate from the study of our Crab spectrum, i.e. from a source which is not in a crowded field as opposed to many of the X-ray binaries studied in this work. We need to verify to which extent our analysis is subject to additional systematic errors when applied to sources in crowded fields. In order to assess this possibility, we have randomly added a source position "Controll" to the input catalog for one of our most complex fields (dataset 9) near GX 354-0 and 4U 1700-377 (see Table 1). This position constitutes the worst case for our analysis as it is close to variable bright sources in a location in the galactic plane less than $15^{\circ}$ from the galactic center where also significant diffuse emission is present. Furthermore, it is about 10 arcmin away from 1RXS J171824.2-402934, an X-ray burster capable of very rare 4 minute bursts with peak intensities up to $1.3 \mathrm{Crab}$ and a thermal spectrum peaking at $5 \mathrm{keV}$ detectable up to $28 \mathrm{keV}$ (Kaptein et al. 2007). As a control case, this position therefore also gives an estimation of the influence of variable and on average faint sources which are not included in our input catalog.

The resulting SPI spectrum (Fig. 22a) does not contain any point with a flux significantly different from zero. All points have less than $2 \sigma$ significance. The only indication of any systematic signal which could fake the presence of a source is in the first bin near $30 \mathrm{keV}$ where a level of $3 \mathrm{mCrab}$ is reached. If we naively fit the points with a powerlaw, we obtain a very steep spectrum with best fit parameters $\Gamma=5.5$, Norm $=2075$.

Repeating the same procedure for the corresponding ISGRI spectrum (Fig. 22b) results in best fit parameters $\Gamma=1.0$, Norm $=1.3 \times 10^{-5}$. Also here all points are consistent with zero. The most significant point is near $300 \mathrm{keV}$ and has $2 \sigma$ with a nominal flux of approx. $80 \mathrm{mCrab}$.

The fluxes derived from these powerlaws can serve as additional estimations of systematic errors on the flux values measured by us. They are given in Table 5 in the row labelled "Estim. Syst. Error" with negative sign in order to indicate that the
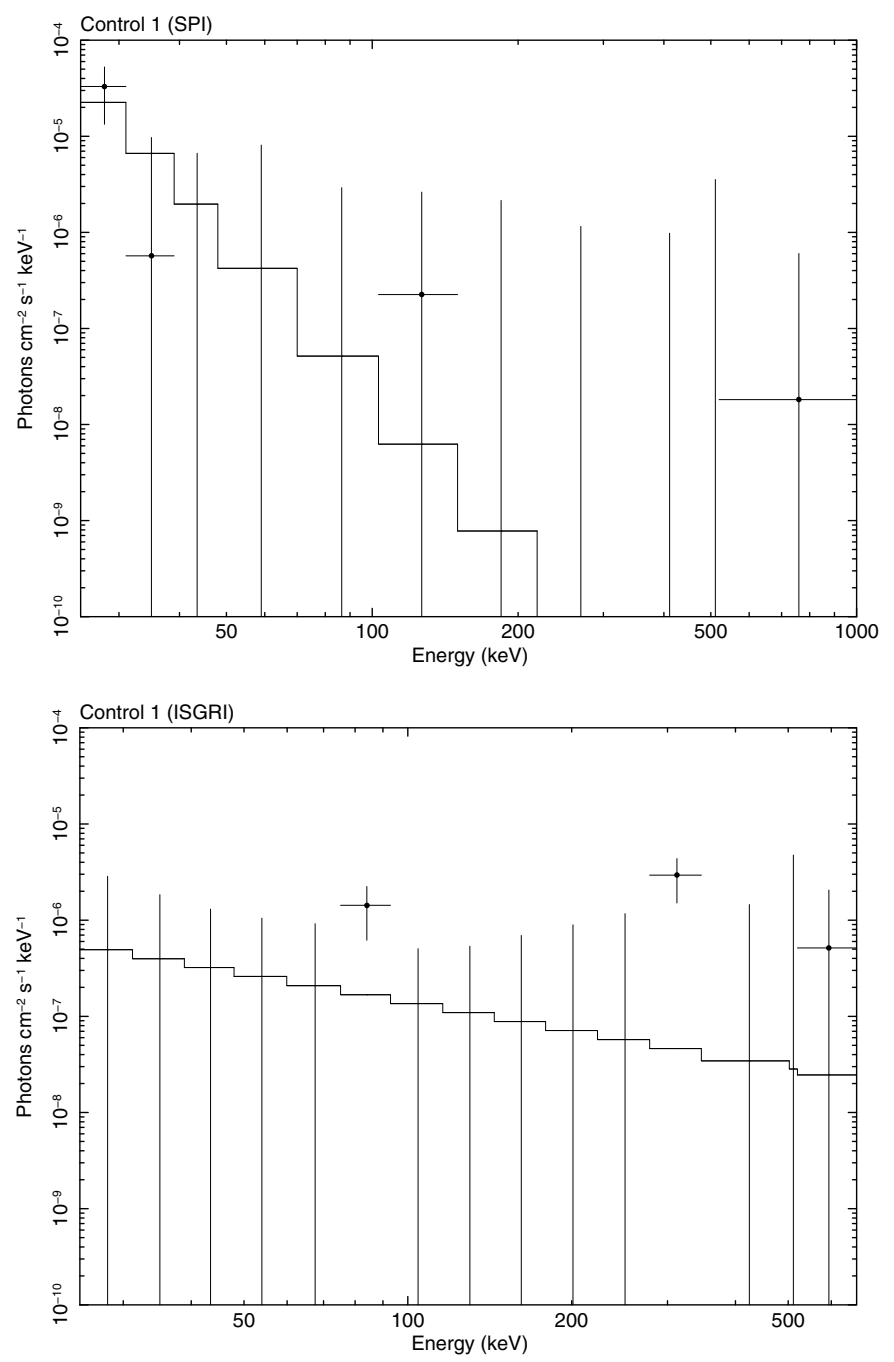

Fig. 22. The unfolded spectra for the control position "Control1" at $\mathrm{RA}=259.5^{\circ}, \mathrm{Dec}=-40.35^{\circ}$ derived from dataset 9 (see Table 1 ) with statistical errors as for all other sources: a) (top) SPI spectrum with powerlaw fit; b) (bottom) ISGRI spectrum with powerlaw fit. The fit parameters are given in Sect. 4.21.

values need to be subtracted from the other flux measurements in the table.

\section{Summary and conclusions}

Tables 2-5 summarise our spectral measurements. They provide a catalog of the time-averaged spectra of the brightest known soft gamma-ray sources. They also give an impression of the variety of possible spectral shapes for different source types, especially for X-ray binaries (XRBs) which represent more than half of the sources. Furthermore, the tables permit to compare the response of SPI and ISGRI. In particular Tables 2 and 3 permit to study the agreement of the corresponding measurements in terms of spectral shape while Table 5 can be used to compare the flux normalisation of the two instruments in several energy bands. In the latter table we also give the results from B08 for those energy ranges where they are available. 
Table 2. Spectral fit parameters for sources other than X-ray binaries.

\begin{tabular}{|c|c|c|c|c|c|c|c|}
\hline \multirow[t]{2}{*}{ Object } & \multirow{2}{*}{$\begin{array}{l}\text { Instru- } \\
\text { ment }\end{array}$} & \multicolumn{6}{|c|}{ 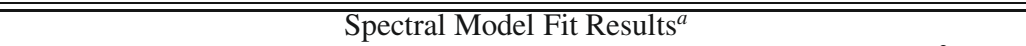 } \\
\hline & & $\Gamma_{1}$ & $\begin{array}{l}E_{\text {cutoff }} \\
{[\mathrm{keV}]}\end{array}$ & $\begin{array}{l}E_{\text {break }} \\
{[\mathrm{keV}]}\end{array}$ & $\Gamma_{2}$ & $\begin{array}{c}\text { Norm } \\
{\left[\mathrm{keV}^{-1} \mathrm{~cm}^{-2} \mathrm{~s}^{-1}\right]}\end{array}$ & $\chi^{2} /$ d.o.f. \\
\hline \multirow{2}{*}{ Crab } & SPI & $2.11 \pm 0.01$ & - & $81 \pm 11$ & $2.20 \pm 0.01$ & $11.6 \pm 0.5$ & 0.5 \\
\hline & ISGRI & $2.119 \pm 0.006$ & - & $96 \pm 4$ & $2.36 \pm 0.02$ & $10.7 \pm 0.3$ & 1.2 \\
\hline \multirow{2}{*}{ Vela Pulsar } & SPI & $2.13 \pm 0.10$ & - & - & - & $0.12 \pm 0.05$ & 0.55 \\
\hline & ISGRI & $2.04 \pm 0.04$ & - & - & - & $0.056 \pm 0.008$ & 1.4 \\
\hline \multirow{2}{*}{ NGC 4151} & SPI & $2.10 \pm 0.17$ & - & - & - & $0.4 \pm 0.25$ & 1.4 \\
\hline & ISGRI & $2.03 \pm 0.06$ & - & - & - & $0.24 \pm 0.05$ & 1.4 \\
\hline \multirow{2}{*}{ NGC 4945} & $\mathrm{SPI}^{b}$ & $1.7 \pm 0.17$ & 150 & - & - & $0.06 \pm 0.04$ & 0.4 \\
\hline & ISGRI $^{b}$ & $1.56 \pm 0.05$ & 150 & - & - & $0.033 \pm 0.006$ & 0.6 \\
\hline \multirow{2}{*}{ Cen A } & SPI & $1.89 \pm 0.05$ & - & - & - & $0.20 \pm 0.04$ & 1.2 \\
\hline & ISGRI & $1.80 \pm 0.02$ & - & - & - & $0.117 \pm 0.009$ & 0.9 \\
\hline \multirow{2}{*}{ Swift J1656.3-3302 } & SPI & $1.5 \pm 0.2$ & - & - & - & $0.004 \pm 0.004$ & 1.0 \\
\hline & ISGRI $^{c}$ & - & - & - & - & - & - \\
\hline
\end{tabular}

${ }^{a}$ Spectral parameters not used in the model for a particular object are marked as "-"; ${ }^{b}$ fit model also included the strong photoelectric absorption with $N_{\mathrm{H}}$ fixed to $5.5 \times 10^{24} \mathrm{~cm}^{-2} ;{ }^{c}$ ISGRI spectral fit failed, see Sect. 4.8 .

Table 3. Spectral fit parameters for X-ray binaries.

\begin{tabular}{|c|c|c|c|c|c|c|c|c|}
\hline \multirow[t]{2}{*}{ Object } & \multirow{2}{*}{$\begin{array}{l}\text { Instru- } \\
\text { ment }\end{array}$} & \multicolumn{7}{|c|}{ 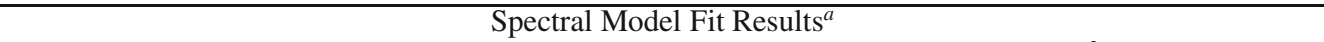 } \\
\hline & & $\Gamma_{1}$ & $\begin{array}{l}E_{\text {cutoff }} \\
{[\mathrm{keV}]}\end{array}$ & $\Gamma_{2}$ & $\begin{array}{c}\text { Norm } \\
{\left[\mathrm{keV}^{-1} \mathrm{~cm}^{-2} \mathrm{~s}^{-1}\right]}\end{array}$ & $\begin{array}{c}\text { Norm }_{2} \\
{\left[10^{-6} \mathrm{keV}^{-1} \mathrm{~cm}^{-2} \mathrm{~s}^{-1}\right]}\end{array}$ & $\chi^{2} /$ d.o.f. & $\begin{array}{l}\text { F-test } \\
\text { prob. }{ }^{b}\end{array}$ \\
\hline \multirow{2}{*}{ XTE J1550-564 ${ }^{c}$} & SPI & $1.36 \pm 0.09$ & $206 \pm 50$ & - & $0.031 \pm 0.010$ & - & 1.6 & - \\
\hline & ISGRI & $1.00 \pm 0.05$ & $108 \pm 9$ & - & $0.009 \pm 0.002$ & - & 1.3 & - \\
\hline \multirow{2}{*}{ 4U 1630-47 } & SPI & $3.3 \pm 0.2$ & - & - & $33 \pm 21$ & - & 0.6 & - \\
\hline & ISGRI $^{d}$ & $2.3 \pm 0.2$ & - & - & $0.043 \pm 0.038$ & - & 1.0 & - \\
\hline \multirow{2}{*}{ OAO $1657-415^{c}$} & SPI & $1.23 \pm 0.07$ & $23.9 \pm 3.2$ & $1.27 \pm 0.58$ & $0.16 \pm 0.04$ & $2.1 \pm 1.0$ & 0.7 & 0.015 \\
\hline & ISGRI & $1.42 \pm 0.31$ & $24.7 \pm 3.6$ & - & $0.23 \pm 0.21$ & - & 0.4 & - \\
\hline \multirow{2}{*}{ GX $339-4^{c}$} & SPI & $1.58 \pm 0.11$ & 100 & $0.97 \pm 0.63$ & $0.081 \pm 0.026$ & $3.8 \pm 2.4$ & 0.8 & 0.0035 \\
\hline & ISGRI & $1.71 \pm 0.10$ & 100 & 1.0 & $0.038 \pm 0.011$ & $1.9 \pm 0.8$ & 0.7 & - \\
\hline \multirow{2}{*}{$4 \mathrm{U} 1700-377^{c}$} & SPI & $2.00 \pm 0.08$ & $47.5 \pm 3.9$ & - & $3.14 \pm 0.71$ & - & 1.6 & - \\
\hline & ISGRI & $2.31 \pm 0.17$ & $74.1 \pm 14.1$ & - & $6.4 \pm 3.2$ & - & 0.9 & - \\
\hline \multirow{2}{*}{ IGR J17091-3624 } & SPI & $1.75 \pm 0.20$ & - & - & $0.016 \pm 0.013$ & - & 0.4 & - \\
\hline & ISGRI & $1.93 \pm 0.04$ & - & - & $0.034 \pm 0.005$ & - & 1.2 & - \\
\hline \multirow{2}{*}{ GX 354- $0^{c}$} & SPI & $3.26 \pm 0.06$ & - & - & $26.4 \pm 5.9$ & $\begin{array}{ll}- & \\
\end{array}$ & 0.7 & - \\
\hline & ISGRI & $3.53 \pm 0.02$ & - & - & $43.3 \pm 3.0$ & - & 1.0 & - \\
\hline \multirow{2}{*}{ 1E $1740.7-2942^{c}$} & SPI & $1.80 \pm 0.06$ & 113.4 & - & $0.28 \pm 0.06$ & $\begin{array}{ll}- \\
-\end{array}$ & 1.9 & - \\
\hline & ISGRI & $1.44 \pm 0.04$ & $113.4 \pm 7.6$ & - & $0.040 \pm 0.004$ & - & 0.7 & - \\
\hline \multirow{2}{*}{ IGR J17464-3213 } & SPI & $2.82 \pm 0.14$ & - & - & $2.1 \pm 1.1$ & - & 1.1 & - \\
\hline & ISGRI & $2.49 \pm 0.02$ & - & - & $0.63 \pm 0.04$ & - & 0.9 & - \\
\hline \multirow{2}{*}{ GRS $1758-258^{c}$} & SPI & $1.54 \pm 0.07$ & $178 \pm 28$ & - & $0.12 \pm 0.027$ & - & 1.7 & - \\
\hline & ISGRI & $1.36 \pm 0.02$ & $123 \pm 5$ & - & $0.06 \pm 0.004$ & - & 1.9 & - \\
\hline \multirow{2}{*}{ Ginga $1826-24^{c}$} & SPI & $1.51 \pm 0.14$ & $48.4 \pm 7.8$ & $0.82 \pm 0.69$ & $0.24 \pm 0.09$ & $2.1 \pm 1.8$ & 0.5 & 0.002 \\
\hline & ISGRI & $1.78 \pm 0.04$ & $68.5 \pm 3.5$ & - & $0.42 \pm 0.05$ & - & 1.5 & - \\
\hline \multirow{2}{*}{ GRS $1915+105^{c}$} & SPI & $1.7 \pm 1.7$ & $12.5 \pm 9.2$ & $2.76 \pm 0.18$ & $4 \pm 15$ & $1.4 \pm 0.1$ & 0.6 & $9.2 \times 10^{-5}$ \\
\hline & ISGRI & $0.3 \pm 1.1$ & $7.6 \pm 2.1$ & $2.87 \pm 0.05$ & $0.09 \pm 0.25$ & $1.17 \pm 0.04$ & 0.7 & $7.8 \times 10^{-9}$ \\
\hline \multirow{2}{*}{ Cyg X-1 ${ }^{c}$} & SPI & $1.28 \pm 0.19$ & $95.9 \pm 15.5$ & $2.11 \pm 0.09$ & $0.43 \pm 0.35$ & $99 \pm 18$ & 0.9 & $9.4 \times 10^{-6}$ \\
\hline & ISGRI & $1.44 \pm 0.14$ & $115 \pm 18$ & $2.20 \pm 0.14$ & $0.90 \pm 0.53$ & $36 \pm 23$ & 1.5 & - \\
\hline \multirow{2}{*}{ Cyg X-3 $3^{c}$} & SPI & $2.0 \pm 0.7$ & $20 \pm 10$ & $2.4 \pm 0.3$ & $3.5 \pm 6.6$ & $9.0 \pm 1.1$ & 0.8 & $3.5 \times 10^{-3}$ \\
\hline & ISGRI & $2.00 \pm 0.08$ & 20 & $2.6 \pm 0.2$ & $3.4 \pm 0.7$ & $5.3 \pm 0.5$ & 1.7 & - \\
\hline
\end{tabular}

${ }^{a}$ Spectral parameters not used in the model for a particular object are marked as "-".

${ }^{b}$ F-test probability that the fit improvement achieved by the inclusion of a hard tail in addition to a cutoff powerlaw was by chance, i.e. that the hard tail is not real. Note that since ISGRI has lower sensitivity above $500 \mathrm{keV}$ and none above $700 \mathrm{keV}$, we can in most cases not test for the presence of the additional component but only check the compatibility with the ISGRI data.

${ }^{c}$ Parameters of alternative fit models are given in Sect. 4.

${ }^{d}$ Spectrum very probably flawed by source contamination from IGR J16358-472.

\subsection{Agreement of SPI and ISGRI spectra}

The study of 20 bright sources with SPI and ISGRI using nearly identical datasets permits us to carry out a quantitative comparison of the response of the two instruments and test the quality of the spectra obtained with our new spimodfit-based analysis method.

The comparison of the absolute flux normalisation should be carried out using a steady source such as the Crab. Our Crab flux measurements are given in Table 4. While the flux ratio $\mathrm{SPI} / \mathrm{ISGRI}$ is consistent with a constant value of about 1.12 up to 
Table 4. Our measurements of the Crab flux with SPI and ISGRI assuming the spectral shape given in Table 2 for energy redistribution.

\begin{tabular}{l|ccccc}
\hline \hline \multirow{2}{*}{ Instrument } & \multicolumn{5}{|c}{ Crab Nebula Flux $\left[10^{-3} \mathrm{~cm}^{-2} \mathrm{~s}^{-1}\right]$} \\
& $25-50 \mathrm{keV}$ & $50-100 \mathrm{keV}$ & $100-200 \mathrm{keV}$ & $200-600 \mathrm{keV}$ & $600-1000 \mathrm{keV}$ \\
\hline SPI & $156.5 \pm 0.4$ & $72.18 \pm 0.15$ & $32.14 \pm 0.12$ & $17.81 \pm 0.27$ & $3.47 \pm 0.15$ \\
ISGRI & $140.2 \pm 0.4$ & $64.38 \pm 0.16$ & $27.46 \pm 0.09$ & $13.47 \pm 0.25$ & - \\
\hline Ratio SPI/ISGRI & $1.116 \pm 0.004$ & $1.121 \pm 0.004$ & $1.170 \pm 0.006$ & $1.322 \pm 0.032$ & - \\
\hline
\end{tabular}

Table 5. Source fluxes [mCrab].

\begin{tabular}{|c|c|c|c|c|c|c|c|c|c|c|}
\hline \multirow[t]{3}{*}{ Object Name } & \multicolumn{10}{|c|}{ Flux [mCrab] $]^{a, b}$} \\
\hline & \multicolumn{3}{|c|}{$25-100 \mathrm{keV}$} & \multicolumn{3}{|c|}{$100-200 \mathrm{keV}$} & \multicolumn{3}{|c|}{$200-600 \mathrm{keV}$} & \multirow{2}{*}{$\begin{array}{c}600-1000 \mathrm{keV} \\
\text { SPI }\end{array}$} \\
\hline & SPI & ISGRI & SPI(B08) & SPI & ISGRI & SPI(B08) & SPI & ISGRI & SPI(B08) & \\
\hline Crab & $1000.0 \pm 2.0$ & $1000.0 \pm 2.0$ & $1000.0 \pm 2.0$ & $1000.0 \pm 3.4$ & $1000.0 \pm 3.5$ & $1000.0 \pm 3.7$ & $1000.0 \pm 11.8$ & $1000.0 \pm 18.6$ & $1000.0 \pm 8.3$ & $1000.0 \pm 43.2$ \\
\hline Vela Pulsar & $10.0 \pm 0.4$ & $7.2 \pm 0.1$ & $9.8 \pm 0.7$ & $11.6 \pm 1.3$ & $10.2 \pm 0.9$ & $12.6 \pm 2.1$ & $5.6 \pm 5.3$ & $0.0+19.0$ & $13.9 \pm 4.9$ & $23.0 \pm 18.5$ \\
\hline NGC 4151 & $35.4 \pm 2.4$ & $32.7 \pm 0.7$ & $31.3 \pm 2.1$ & $43.8 \pm 7.4$ & $34.6 \pm 2.5$ & $22.9 \pm 6.8$ & $28.2 \pm 36.4$ & $0.0+132.5$ & $46.9 \pm 14.1$ & $101.4 \pm 111$ \\
\hline NGC 4 & $18.7 \pm 1.1$ & $16.4 \pm 0.3$ & $18.3 \pm 1.1$ & $21.4 \pm 3.7$ & $21.5 \pm 1.2$ & $14.3 \pm 4.0$ & $0.5 \pm 27.1$ & $0.0+53.2$ & $20.2 \pm 8.3$ & $5.8 \pm 53.8$ \\
\hline Cen A & $40.7 \pm 1.1$ & $36.0 \pm 0.3$ & $40.5 \pm 1.3$ & $59.7 \pm 3.7$ & $53.7 \pm 1.2$ & $56.9 \pm 4.6$ & $55.9 \pm 20.7$ & $87.7 \pm 24.2$ & $0.1 \pm 9.8$ & $12.9 \pm 48.7$ \\
\hline XTE J15 & $36.1 \pm 0.6$ & $41.6 \pm 0.3$ & $23.5 \pm 0.7$ & $55.2 \pm 1.7$ & $70.2 \pm 0.9$ & $30.2 \pm 2.5$ & $48.8 \pm 8.4$ & $51.5 \pm 12.1$ & $18.8 \pm 5.5$ & $6.7 \pm 24.4$ \\
\hline $4 \mathrm{U} 16$ & $36.5 \pm 1.6$ & $1.8 \pm 0.2^{c}$ & $30.5 \pm 1.3$ & $19.7 \pm 6.0$ & $0.8 \pm 0.3^{c}$ & $21.7 \pm 2.4$ & $2.2 \pm 23.3$ & $19.9 \pm 13.2^{c}$ & 5.3 & $0.0+164.2$ \\
\hline Swift & $3.7 \pm 2.2$ & $-d$ & $6.0 \pm 1.1$ & $8.0 \pm 3.4$ & $-d$ & $5.0 \pm 1.9$ & $15.2 \pm 7.8$ & $-d$ & \pm 4.0 & $24.6 \pm 12.9$ \\
\hline OAO 1 & $66.6 \pm 0.6$ & $51.5 \pm 1.3$ & $51.3 \pm 0.7$ & $15.0 \pm 1.3$ & $7.6 \pm 0.6$ & $10.3 \pm 2.2$ & $15.0 \pm 8.0$ & $0.0+24.9$ & $13.8 \pm 4.8$ & $2.3 \pm 26.6$ \\
\hline GX 339-4 & $34.1 \pm 0.5$ & $11.5 \pm 0.2$ & $38.4 \pm 1.4$ & $32.6 \pm 1.4$ & $12.1 \pm 0.5$ & $35.6 \pm 2.4$ & $30.1 \pm 13.8$ & $0.0+30.3$ & $30.0 \pm 5.2$ & $66.1 \pm 27.4$ \\
\hline $4 \mathrm{U} 17$ & $171.7 \pm 0.8$ & $162.3 \pm 3.6$ & $146.3 \pm 1.4$ & $36.0 \pm 1.0$ & $40.9 \pm 1.6$ & $35.5 \pm 2.2$ & $13.6 \pm 8.1$ & $23.4 \pm 17.2$ & $6.4 \pm 4.8$ & $0.0+62.7$ \\
\hline IGR J1 & $5.2 \pm 0.8$ & $6.1 \pm 0.1$ & $8.9 \pm 0.6$ & $7.2 \pm 5.0$ & $6.3 \pm 0.3$ & $5.6 \pm 2.0$ & $3.5 \pm 8.8$ & $2.7 \pm 13.4$ & $9.8 \pm 4.4$ & $8.7 \pm 19.6$ \\
\hline GX & \pm 0.5 & $23.4 \pm 0.1$ & 0.5 & 1.1 & $5.4 \pm 0.2$ & 1.8 & 0.0 & 1.0 & & 1.4 \\
\hline 1E 1 & $8 \pm 0.9$ & $32.7 \pm 0.1$ & $51.1 \pm 1.2$ & $43.0 \pm 2.3$ & $35.0 \pm 0.3$ & $48.3 \pm 1.6$ & $18.5 \pm$ & $22.1 \pm 6.1$ & $39.2 \pm 3.6$ & $7.8 \pm 34.9$ \\
\hline IGR J & $13.3 \pm 0.6$ & $15.1 \pm 0.1$ & $15.6 \pm 0.7$ & $6.9 \pm 1.1$ & $9.9 \pm 0.2$ & $11.2 \pm 1.6$ & $2.2 \pm 4.4$ & $6.8 \pm 6.3$ & $9.9 \pm 3.6$ & $41.6 \pm 23.1$ \\
\hline GRS & $68.8 \pm 0.7$ & $66.6 \pm 0.2$ & $70.8 \pm 0.9$ & $84.6 \pm 2.1$ & $82.2 \pm 0.5$ & $83.4 \pm 1.6$ & $41.1 \pm 7.2$ & $49.3 \pm 6.0$ & $47.4 \pm 3.6$ & $51.2 \pm 18.0$ \\
\hline Ginga 1826-24 & $75.8 \pm 0.5$ & $73.1 \pm 0.3$ & $74.6 \pm 0.5$ & $30.3 \pm 0.9$ & $34.2 \pm 0.4$ & $33.6 \pm 1.9$ & $18.4 \pm 5.7$ & $0.0+19.8$ & $24.5 \pm 3.9$ & $38.3 \pm 22.4$ \\
\hline GRS $1915+105$ & $189.1 \pm 0.8$ & $187.8 \pm 0.5$ & $150.1 \pm 0.9$ & $73.2 \pm 2.2$ & $60.5 \pm 0.5$ & $61.8 \pm 2.3$ & $38.8 \pm 8.7$ & $40.9 \pm 10.2$ & $38.6 \pm 5.2$ & $53.9 \pm 27.2$ \\
\hline Cyg X-1 & $841.0 \pm 1.5$ & $871.6 \pm 1.7$ & $843.8 \pm 1.0$ & $873.7 \pm 3.1$ & $907.6 \pm 3.1$ & $769.9 \pm 3.1$ & $575.5 \pm 9.8$ & $581.3 \pm 16.1$ & $500.6 \pm 6.9$ & $386.4 \pm 37.3$ \\
\hline Cyg X-3 & $117.7 \pm 0.6$ & $111.7 \pm 0.6$ & $101.2 \pm 0.8$ & $34.9 \pm 1.4$ & $25.3 \pm 0.5$ & $25.0 \pm 2.9$ & $26.4 \pm 7.6$ & $21.0 \pm 21.8$ & $14.8 \pm 6.2$ & $52.6 \pm 31.0$ \\
\hline Estim. Syst. Error ${ }^{e}$ & -0.9 & -0.1 & - & -0.1 & -0.4 & - & -0.1 & -1.2 & - & -0.1 \\
\hline
\end{tabular}

${ }^{a}$ For the columns "SPI" and "ISGRI" fluxes are expressed as fractions of the Crab fluxes for the individual instrument given in Table 4. In the columns "SPI (B08)" we give the fluxes obtained by Bouchet et al. (2008) (B08) for the available energy ranges (see B08 for their definition of the unit "mCrab").

${ }^{b}$ The statistical errors shown in this table do not take into account the uncertainty of the Crab flux on which the flux values are normalised.

${ }^{c}$ Spectrum very probably flawed by source contamination from IGR J16358-472; therefore not included in Fig. 23.

${ }^{d}$ ISGRI spectral fit failed, see Sect. 4.8; therefore not included in Fig. 23.

${ }^{e}$ See Sect. 4.21 .

$200 \mathrm{keV}$, we observe a clear deviation at higher energies suggesting systematic differences in flux calibration of additional $18 \%$ at 200-600 keV (see Sect. 4.1).

If we correct for the differences in absolute flux calibration by normalising on the Crab flux, we obtain the flux values in Table 5. Plotting the SPI values versus the ISGRI values from this table, we obtain Figs. 23a-c. In all three energy ranges up to $600 \mathrm{keV}$, these plots show clearly a good agreement between the flux values from the two instruments for all sources with fluxes higher than a few ten mCrab. Only for the faintest sources and only below $100 \mathrm{keV}$ do we see obvious systematic deviations from the linear relationship. Here the SPI fluxes tend to be lower than those from ISGRI. The origin of this is probably a combination of two causes: (1) systematic differences in the handling of source variability by the analysis software; and (2) systematic differences in the background determination.

\subsection{Agreement of our SPI measurements with those by B08}

To further verify our analysis method, we compare our SPI fluxes with those published by B08 who give their flux values as fraction of their Crab flux. All values are given in Table 5. Plotting our values versus those from B08 (Figs. 24a-c) we see that the flux values agree well in all energy bands for sources brighter than $20 \mathrm{mCrab}$. Below this flux level, our flux values seem to be systematically slightly lower. At least some of the differences can be attributed to source variability as our dataset is not exactly the same as that used by B08.

B08 defined the annihilation (positronium) emission between $300 \mathrm{keV}$ and $511 \mathrm{keV}$ as an individual component in their source model and extracted its intensity as a result of their fit. Since this emission is extended (to first order a $8^{\circ} \mathrm{FWHM}$ Gaussian centered on the galactic centre) on a scale similar to the SPI field-of-view, it is in our analysis absorbed in the timevariable isotropic component of our background model which does not have a separate annihilation component. This is confirmed by the fact that our fluxes in the range $200-600 \mathrm{keV}$ agree very well with those by B08 (see Fig. 24), also for sources near the galactic centre.

\subsection{Emission above $200 \mathrm{keV}$}

We detect all of the 20 sources in our sample independently in the $25-100 \mathrm{keV}$ and in the $100-200 \mathrm{keV}$ band both with SPI and with ISGRI. Above $200 \mathrm{keV}$ we find evidence for emission 

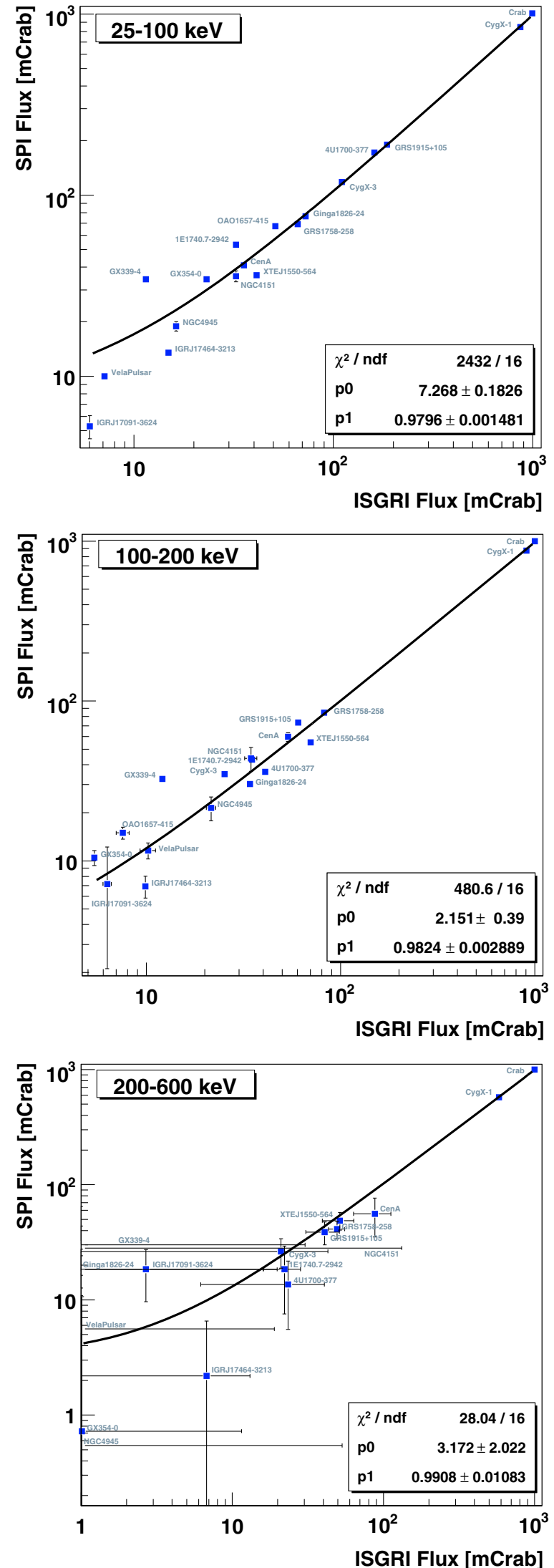

Fig. 23. Comparison of our flux values obtained from SPI with those from ISGRI for the energy bands a) (top) $25-100 \mathrm{keV}$; b) (middle) $100-200 \mathrm{keV}$; c) (bottom) 200-600 keV. In each band, a linear function was fit to the points and fit parameters are shown in the figures. The flux values are also given in Table 5. Only the statistical errors are shown. They take into account the uncertainty of the Crab flux. See Sect. 5.1.
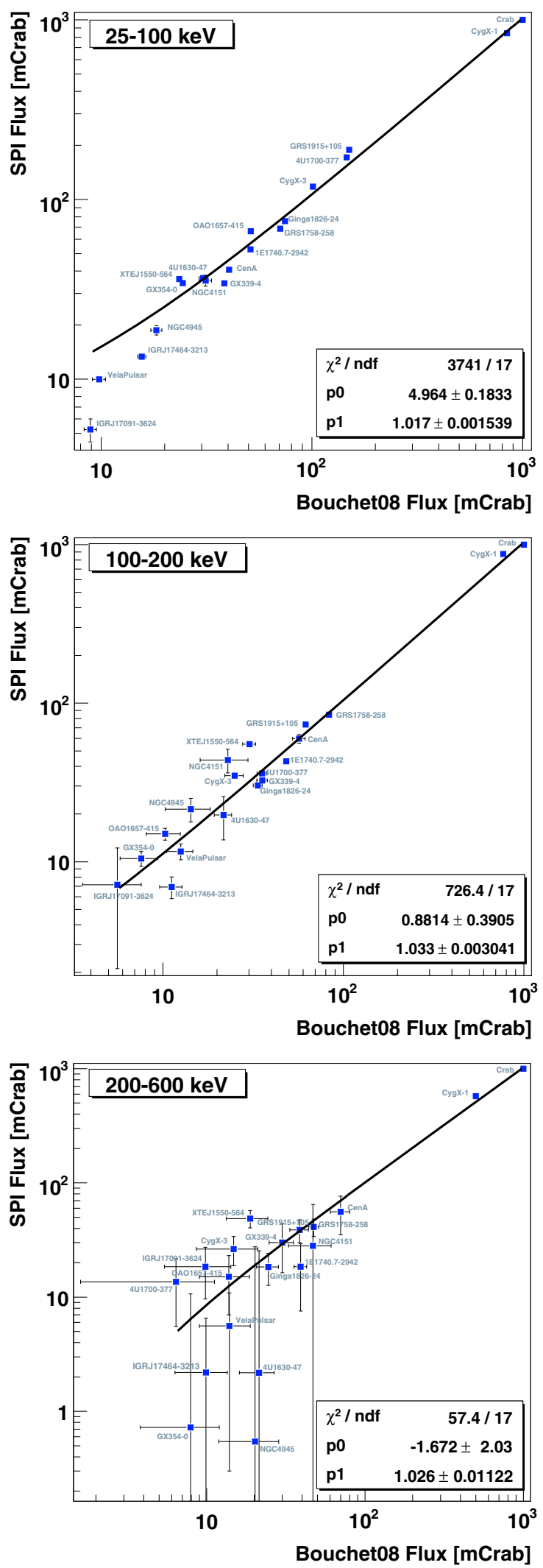

Fig. 24. Comparison of our flux values obtained from SPI with those by B08 (also obtained with SPI, largely using the same data but with a different analysis method) for the energy bands a) (top) $25-100 \mathrm{keV}$; b) (middle) 100-200 keV; c) (bottom) 200-600 keV. In each band, a linear function was fit to the points and fit parameters are shown in the figures. The flux values are also given in Table 5. Only the statistical errors are shown. They take into account the uncertainty of the Crab flux. See Sect. 5.2. 
of more than $3 \sigma$ in 8 cases. In the $600-1000 \mathrm{keV}$ band, we find such evidence only for two sources.

XRBs constitute $70 \%$ of our source sample. Table 3 summarises our measurements of the spectra of these 14 XRBs. They are not a random sample but the 14 brightest known X-ray binaries at $200 \mathrm{keV}$. Four of them are HMXBs, the remaining are LMXBs. Ten of them contain black hole candidates (eight of which are classified as microquasars), the remaining four contain neutron stars.

\subsection{Conclusions}

We present spectra for the 20 brightest soft gamma-ray sources averaged over timescales of years and give flux values in four energy bands between $25 \mathrm{keV}$ and $1 \mathrm{MeV}$.

For SPI, these measurements were derived using a new tool (spimodift by Halloin \& Strong 2007) which can coherently treat data from the whole SPI mission by performing a single, large maximum likelihood fit.

The agreement between our SPI and ISGRI measurements is good if we correct for systematic calibration differences by normalizing on the Crab spectrum. Our SPI flux measurements also agree well with those by Bouchet et al. (2008) (B08). Our Crab spectrum shows the also previously observed break near $100 \mathrm{keV}$ where the spectral index softens from 2.1 to 2.2.

Up to $200 \mathrm{keV}$, all 20 sources in our sample are detected independently in two adjacent bands. At 200-600 keV we detect eight sources, and at $600-1000 \mathrm{keV}$ we detect two sources. The spectra we find agree well with the results from previous publications. For six of the 14 XRBs in our sample (not counting the borderline case GRS 1758-258), we find evidence for a hard powerlaw-component which becomes dominant above the cutoff energy of the thermal Comptonization component, i.e. above about hundred to a few hundred keV. For GX 339-4, GRS 1915+105, Cyg X-1, and Cyg X-3 such hard tails have already been noted by previous studies. For OAO 1657-415 and Ginga 1826-24, our study provides the first weak indication of such emission.

Acknowledgements. We would like to thank R. Diehl, J. Greiner, and G. Sala (MPE, Garching), and E. Jourdain and J. P. Roques (CESR/CNRS, Toulouse) for useful discussions. D.P. was supported in part by the German Bundesministerium für Bildung, Wissenschaft, Forschung und Technologie (BMBF/DLR) under contract No. FKZ 50 OG 0502.

\section{References}

Alvarez-Gaumé, L., et al. 2004, Rev. Particle Phys., Phys. Lett. B, 592 Arnaud, K., Dorman, B., \& Gordon, C. 2007, Xspec - an X-ray fitting package, User's Guide for version 12.3.1, HEASARC, NASA/GSFC publications Audley, M. D., Nagase, F., Mitsuda, K., et al. 2006, MNRAS, 367, 1147 Barnstedt, J., Staubert, R., Santangelo, A., et al. 2008, A\&A, 486, 293 Beckmann, V., Shrader, C. R., Gehrels, N., et al. 2005, ApJ, 634, 939 Bosch-Ramon, V., Romero, G. E., Paredes, J. M., et al. 2006, A\&A, 457, 1011

Bouchet, L., Mandrou, P., Roques, J. P., et al. 1991, ApJ, 383, L45 Bouchet, L., Roques, J. P., Mandrou, P., et al. 2005, ApJ, 635, 1115 Bouchet, L., Jourdain, E., Roques, J. P., et al. 2008, ApJ, 679, 1315 Cadolle Bel, M., Sizun, P., Goldwurm, A., et al. 2006, A\&A, 446, 591 Capitanio, F., Bazzano, A., Ubertini, P., et al. 2006a, Adv. Space Res., 38, 2816

Capitanio, F., Bazzano, A., Ubertini, P., et al. 2006b, ApJ, 643, 376

Chaty, S., Rahoui, F., Foellmi, C., et al. 2008, A\&A, 484, 783

Corbel, S., Kaaret, P., Fender, R. P., et al. 2005, ApJ, 632, 504

Courvoisier, T. J.-L., Walter, R., Beckmann, V., et al. 2003, A\&A, 411, L53
Courvoisier, T. J.-L., et al. 2008, INTEGRAL Source Results Version 2, available from the INTEGRAL Science Data Centre website

http://isdc.unige.ch/

Del Santo, M., Bazzano, A., Zdziarski, A. A., et al. 2005, A\&A, 433, 613

Dermer, C. D., \& Gehrels, N. 1995, ApJ, 447, 103

Done, C., Madjeski, G. M., \& Smith, D. A. 1996, ApJ, 463, L63

Done, C., Wardzinski, G., \& Gierlinski, M. 2004, MNRAS, 349, 393

Falanga, M., Götz, D., Goldoni, P., et al. 2006, A\&A, 458, 21

Fender, R., \& Belloni, T. 2004, ARA\&A, 42, 317

Filippova, E. V., Tsygankov, S. S., Lutovinov, A. A., \& Sunyaev, R. A. 2005, Astron. Lett., 30, 824

Gies, D. R., Bolton, C. T., Thomson, J. R., et al. 2003, ApJ, 583, 424

Goldwurm, A., David, P., Foschini, L., et al. 2003, A\&A, 411, L223

Grabelsky, D. A., Maltz, S. M., Purcell, W. R., et al. 1995, ApJ, 441, 800

Greiner, J., Morgan, E. H., \& Remillard, R. A. 1996, ApJ, 473, L107

Halloin, H., \& Strong, A., 2007, spimodfit user manual, OSA documentation, available from the INTEGRAL Science Data Centre website http: //isdc. unige.ch/

Hjalmarsdotter, L., Zdziarski, A. A., Larsson, S., et al. 2008, MNRAS, 384, 278 Itoh, T., Done, C., Makishima, K., et al. 2008, Publ. Astron. Soc. Japan, 60, S251 Johnson, W. N., Kurfess, J. D., Purcell, W. R., et al. 1993, A\&AS, 97, 21 Joinet, A., Jourdain, E., Malzac, J., et al. 2005, ApJ, 629, 1008

Joinet, A., Jourdain, E., Malzac, J., et al. 2007, ApJ, 657, 400

Jourdain, E., Gotz, D., Westergaard, N. J., et al. 2008 [arXiv : 0810 . 0646], POS (integral08) 144

Kaptein, R. G., in't Zand, J. J. M., Kuulkers, E., et al. 2000, A\&A, 358, L71

Kirsch, M. G., Briel, U. G., Burrows, D., et al. 2005, in Proc. SPIE 5898, ed. O. H. W. Sigmund et al., 589803-1

Krivonos, R., Revnivtsev, M., Churazov, E., et al. 2007, A\&A, 463, 957

Kubota, A., Dotani, T., Cottam, J., et al. 2007, Publ. Astron. Soc. Japan, 59, S185

Maisack, M., Kendziorra, E., Pan, H. C., et al. 1994, A\&A, 283, 841

Malzac, J., Petrucci, P. O., Jourdain, E., et al. 2006, A\&A, 448, 1125

Mangano, V., Bocchino, F., Cusumano, G., et al. 2006, Adv. Space Res., 37, 1984

Marti, J., Perez-Ramirez, D., Luque-Escamilla, P., et al. 2007, Ap\&SS, 309, 309

Masetti, N., Mason, E., Landi, R., et al. 2008, A\&A, 480, 712

McConnell, M. L., Forrest, D. J., Owens, A., et al. 1989, ApJ, 343, 317

McConnell, M. L., Ryan, J. M., Collmar, W., et al. 2000, ApJ, 543, 928

Okajima, T., Tueller, J., Markwardt, C., et al. 2006, ATel, 799

Pottschmidt, K., Chernyakova, M., Zdziarski, A. A., et al. 2006, A\&A, 452, 285

Revnivtsev, M. G., Chernyakova, M., Capitanio, F., et al. 2003, ATel, 132, 1

Revnivtsev, M. G., Gilfanov, M., Churazov, E., \& Sunyaev, R. 2003, ATel, 150, 1

Revnivtsev, M. G., Sunyaev, R. A., Gilfanov, M. R., et al. 2004, Astron. Lett., 30,527

Revnivtsev, M. G., Sazonov, S., Gilfanov, M., Churazov, E., \& Sunyaev, R. 2006, A\&A, 452, 169

Rodriguez, J., Hannikainen, D. C., Shaw, S. E., et al. 2008, ApJ, 675, 1436

Rodriguez, J., Shaw, S. E., Hannikainen, D. C., et al. 2008, ApJ, 675, 1449

Roques, J. P., Schanne, S., von Kienlin, A., et al. 2003, A\&A, 411, L91

Rothschild, R. E., Wilms, J., Tomsick, J., et al. 2006, ApJ, 641, 801

Russell, D. M., Fender, R. P., Gallo, E., \& Kaiser, C. R. 2007, MNRAS, 376, 1341

Schönfelder, V., Bennett, K., Blom, J. J., et al. 2000, A\&AS, 143, 145

Shaposhnikov, N., \& Titarchuk, L. 2006, ApJ, 643, 1098

Smith, D. A. 1998, IAU Circ., 7008, 1

Steinle, H., Bennett, K., Bloemen, H., et al. 1998, A\&A, 330, 97

Strickman, M., Skibo, J., Purcell, W., Barret, D., \& Motch, C. 1996, A\&AS, 120, 217

Strickman, M., de Jager, O., \& Harding, A. 1996, A\&AS, 120, 449

Sturner, S. J., \& Shrader, C. R. 2005, ApJ, 625, 923

Szostek, A., \& Zdziarski, A. A. 2007, MNRAS, 375, 793

Szostek, A., \& Zdziarski, A. A. 2008, MNRAS, 386, 593

Thompson, T. W. J., Rothschild, R. E., Tomsick, J. A., \& Marshall, H. L. 2005, ApJ, 634, 1261

Tomsick, J. A., Corbel, S., Goldwurm, A., \& Kaaret, P. 2005, ApJ, 630, 413

Tomsick, J. A., Kalemci, E., Kaaret, P., et al. 2008, ApJ, 680, 593

Titarchuk, L. 1994, ApJ, 434, 570

Ubertini, P., Lebrun, F., Di Cocco, G., et al. 2003, A\&A, 411, L131

Ulrich, M. H. 2000, A\&ARv, 10, 135

van der Meer, A., Kaper, L., di Salvo, T., et al. 2005, A\&A, 432, 999

Vedrenne, G., Roques, J.-P., Schönfelder, V., et al. 2003, A\&A, 411, L63

Wu, Y.-X., Liu, C.-Z., \& Li, T.-P. 2007, ApJ, 660, 1386

Zhang, S. N., Cui, W., Harmon, B. A., et al. 1997, ApJ, 477, L95

Ziolkowski, J. 2005, MNRAS, 358, 851 